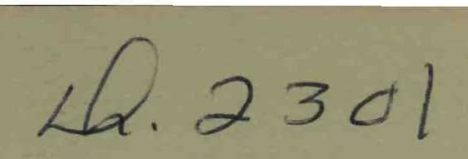

SAND75-0248D

Unlimited Release

UC-71

\title{
Transportation Technical Environmental Information Center Index
}

Clarence A. Davidson, Jerry T. Foley

Prepared by Sandia Laboratories, Albuquerque, New Mexico 87185

and Livermore California 94550 for the United States Danartwan

of Energy under Contract AT(29-1)789

Printed January 1979

Sandia Laboratories 
Issued by Sandia Laboratories, operated for the United States Department of Energy by Sandia Corporation.

\section{NOTICE}

This report was prepared as an account of work sponsored by the United States Government. Neither the United States nor the Department of Energy, nor any of their employees, nor any of their contractors, subcontractors, or their employees, makes any warranty, express or implied, or assumes any legal liability or responsibility for the accuracy, completeness or usefulness of any information, apparatus, product or process disclosed, or represents that its use would not infringe privately owned rights.

Printed in the United States of America

Available from

National Technical Information Service

U. S. Department of Commerce

5285 Port Royal Road

Springfield, VA 22161

Price: Printed Copy $\$ 5.25$; Microfiche $\$ 3.00$ 


\section{DISCLAIMER}

This report was prepared as an account of work sponsored by an agency of the United States Government. Neither the United States Government nor any agency Thereof, nor any of their employees, makes any warranty, express or implied, or assumes any legal liability or responsibility for the accuracy, completeness, or usefulness of any information, apparatus, product, or process disclosed, or represents that its use would not infringe privately owned rights. Reference herein to any specific commercial product, process, or service by trade name, trademark, manufacturer, or otherwise does not necessarily constitute or imply its endorsement, recommendation, or favoring by the United States Government or any agency thereof. The views and opinions of authors expressed herein do not necessarily state or reflect those of the United States Government or any agency thereof. 


\section{DISCLAIMER}

Portions of this document may be illegible in electronic image products. Images are produced from the best available original document. 
SAND75-0248D

Unlimited Release

Printed January 1979

Supersedes SAND75-0248C
Distribution

Category UC-71

\title{
TRANSPORTATION TECHNICAL ENVIRONMENTAL INFORMATION CENTER INDEX
}

Clarence A. Davidson

Jerry T. Foley

Applied Mechanics Division II, 5522

Sandia Laboratories

Albuque r'que, NM 87185

\begin{abstract}
In an effort to determine the environmental intensities to which energy materials in transit may be exposed, a "Data

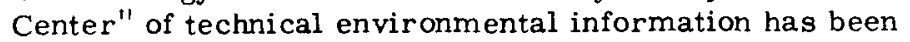
established by Sandia Laboratories, Division 5522, for the DOE Division of Environmental Control Technology. This document is an index which can be used to request data of interest.
\end{abstract}


•

- 
CONTENTS

$\underline{\text { Page }}$

Int roduction

The Environments

The Data

Mechanics of the Data Center

Data Sources

Availability of the Data

The Index

Normal Environments

Abnormal Environments 
0 


\title{
TRANSPORTATION TECHNICAL ENVIRONMENTAL INFORMATION CENTER INDEX
}

\author{
Introduction
}

The purpose of the Transportation Technical Environmental Information Center is to collect, analyze, store, and make available descriptions of the environment of transportation expressed in engineering terms. The data stored in the Center are expected to be useful in a variety of transportation related analyses. Formulations of environmental criteria for shipment of cargo, risk assessments, and detailed structural analyses of shipping containers are examples where these data have been applied. The Transportation Technical Environmental Information Center is a segment of a larger DOE/DOD Environmental Data Center for weapons application.

For purposes of indexing and data retrieval, the data are catalogued under two major headings: Normal and Abnormal Environments. The diagram below illustrates a conceptual relationship between these environmental designations as a function of the expected rate of occurrence.

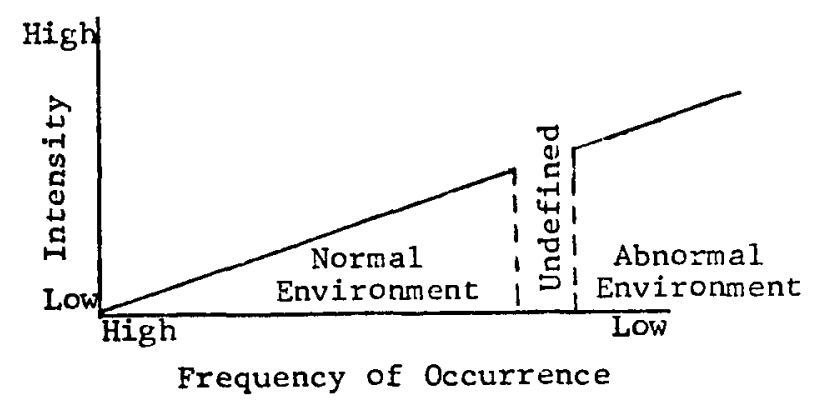

Normal environments are those which will be encountered at some intensity during every shipment. They have a high frequency of occurrence, but a relatively low intensity. The abnormal or accident environments, on the other hand, are characterized by higher intensities but lower frequency of occurrence. The very high intensities of environment occur very infrequently. The abnormal environments, while of ten called by different names to differentiate them, have actually the same engineering parameters as the normal. Thus, an acceleration is experienced by cargo as a truck crosses railroad tracks at $50 \mathrm{mph}$. A greater acceleration may result if the truck strikes a bridge abutment at the same speed. To make the difference clear in a succinct manner, the latter is termed, "impact." These short descriptions are shown in the index in parentheses where they apply. 


\section{The Environments}

T welvo environmental categories have been identified as relevant to transportation. These include:
1. Acceleration/Time
2. Acoustic Noise
3. Atmospheric Contents
4. Fragmentation ${ }^{*}$
5. Humidity
6. Precipitation
7. Pressure
8. Radiation *
9. Shock ${ }^{*}$
10. Temperalure
11. Vibration
12. Wind

Three factors operate to limit the number of these environments for which abnormal levels require consideration:

1. Some environments reach an absolute limit regularly and are totally contained with in a normal range;

2. The abnormal aspects of some environments are encountered so infrequently as to be of little interest; and

3. Protection against the effects of some normal environments include protection against the abnormal

After eliminating those environmental categories covered by the above factors only the environments indicated by an asterisk in the above list are of interest in the abnormal considerations. Figure 1 is a chart which illustrates the organization of data in the Center by categories and by additional nomenclature which differentiates between input and response phenomena. Input may be defined as the environmental level which exists at the cargo interface, while response is measured in terms of how the cargo reacts to the environments. As an example consider the pressure environment, which has both normal and abnormal levels. The normal, which all cargo will experience, includes the variations in atmospheric pressure at a specific location, as well as the variations produced by changes in elevation, both on the earth's surface and while flying. The abnormal aspects of the pressure environment are the result of exposure to an explosion or to immersion (inputs); if the input pressure is sufficiently high, a crush (collapse) response of the cargo will result.

Four of the environments are considered only in the normal aspects for reasons mentioned above. One environment, fragmentation, is considered only in the abnormal aspect. This is because no elements which will cause puncture to the cargo are present under normal conditions, i. e., those which will be encountered frequently during transportation.

\footnotetext{
Lnvironments with both normal and abnormal aspects.
} 


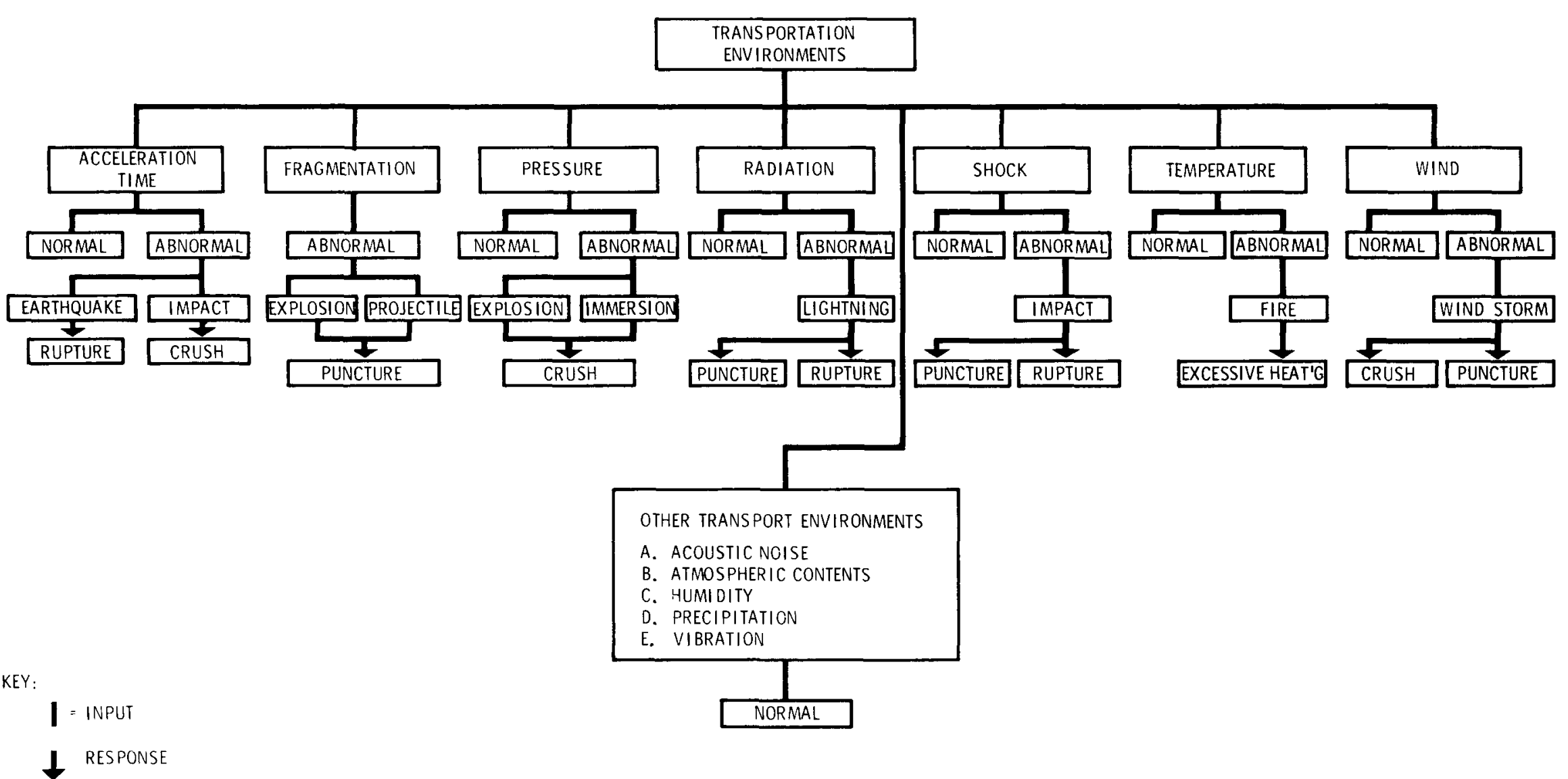

Figure 1. Structure of the Transportation Technical Environmental Information Center 
Normal environmental data stored in the Transportation Technical Environmental Information Center are measured data. They were obtained from reliable sources, both within Sandia Laboratories and from other reputable agencies. When data were required, but not available, the Data Center operators arranged for measurements to provide some of the information.

Most input data in the Data Center under the heading of Abnormal Environments, however, were not measured directly. Because of the number and randomness of occurrence and type, it is impossible to provide engineering instrumentation for measurement. If an instrumented vehicle is subjected to a staged accident, it represents only the one type of event. Therefore, it is necessary to use statistical techniques to characterize the range of environmental intensities which may be experienced by cargo in accidents. The data to which these techniques are applied are often estimated. The basic information which is most useful in estimating abnormal inputs includes the following.

Abnormal Input

1. Earthquake

2. Impact

3. Explosion

4. Projectile/Impalement

5. Immersion

6. Lightning

7. Fire

8. Windstorm
Useful Data Parameters

Time History
Vehicle Velocity
Pressure or Missile Velocity, Radius of
Missile Travel
Package Velocity, Radius of Probe
Depth
Voltage, Amperage, Rise Time, etc
Temperature, Heat Flux and Duration
Velocity, Pressure Differential

It is these types of data, or information which permit the derivation of those values, which are stored in the input section of the $\Lambda$ bnormal Environment part of the Transportation Technical Environmental Information Center. The response section of the Center is expressed in terms of damage to cargo. Since the cargo damage can be described and measured, measurements and descriptions are included wherever possible.

\section{Mechanics of the Data Center}

The data which have been retrieved and entered in the Data Center are on microfilm. Two forms of microfilm are used: aperture cards and microfiche. Facilities for viewing the data and obtaining hard copy are located at the Center site. When processing large requests for information, the Center's operators have access to automated means for obtaining hard copy. 
The two-part number listed after the title of a particular Data Center entry is the access number for the data file. The first number, e.g., 1614, is the file number. The number after the dash (-) is the number of file cards available on the subject. Requests for hard copies of data should cite these two numbers. For large data requests, it is faster and cheaper for the data center operators to transmit the data to the requestor in the form of duplicate microfilm aperture cards or duplicate microfiche. If the requestor has facilities for reading information in these formats, it should be so stated in the request.

When either raw data or published information is acquired, it is reviewed for enginee ring data content. Pertinent information is then extracted, collated, and microfilmed. The data cards and/or microfiche are filed in numerical order without regard to subject. This simple technique is possible because of a computerized indexing and retrieval program whicn was developed for efficient use of the Data Center.

The use of a computer-aided system makes it possible to obtain a more flexible file, permits more complete data retrieval, shortens the time necessary for search, and makes feasible more frequent publication of a current index.

\section{Data Sources}

Normal environmental information for inclusion in the Data Center is acquired by the Data Center's operators from many sources. Military agencies, such as Aberdeen Proving Ground, Wright Air Development Center, Naval Ordnance Test Center, and Army QM R\&E Center, and industrial groups, such as railroads, aircraft companies, and trucking concerns, have contributed information. A considerable portion of the information has been obtained from various project groups within Sandia Laboratories. The Data Center's operators not only extract information from published sources, but engage in specific research activities to obtain data not otherwise available.

Abnormal environmental information is obtained primarily from U. S. Department of Transportation sources and reports produced by the National Transportation Safety Board. Contacts have been established with various individuals and agencies responsible for accident investigation in the various modes of transportation. An information sources file is maintained as an adjunct to the Center which integrates this Data Center's efforts with other Data Banks collecting related accident information such as accident rates, fatalities, etc. 


\section{Availability of the Data}

The information in the Data Center is available, within the limits of time, workload, and security regulations, to any requestor. Requests for data should be sent to Division 5523, Sandia Laboratories, Albuquerque, New Mexico, 87185. The request should either outline the specific area for which information is desired, or it should list specific entries, including serial numbers. Requests will be processed as time allows. Agencies outside of the DOE complex will be furnished information subject to the review and release procedures established by the DOE.

The Index

The index provided on the following pages is what the Center's operators call a category index. Entry is made into this index by finding the environmental category of interest, such as shock, temperature, vibration, etc. The 12 categories are listed alphabetically. 
Normal Environments 


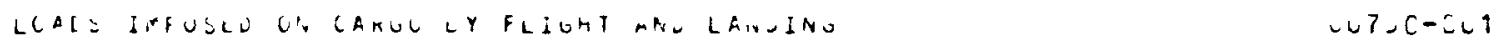
ADELVER LZAD WATA C-1_- -1RCKAFT

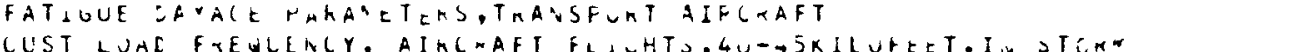

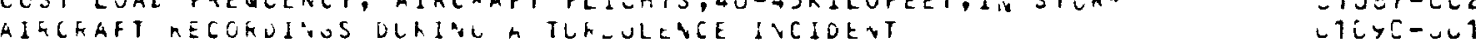

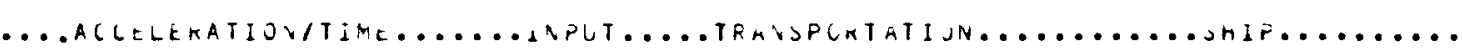

ACCELERATIUPOSBIF NOTIUNS, MAX MLE ACLELEFATJUN,EAFECTEV FEAK VALUE, NARIVE

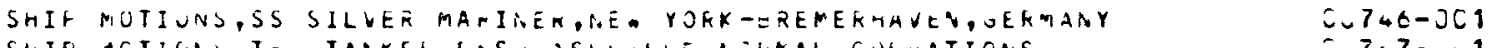

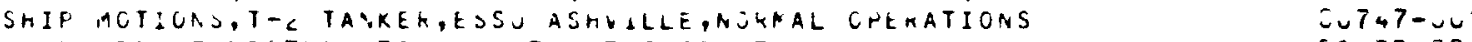

$C_{1}>73-3 C_{4}$

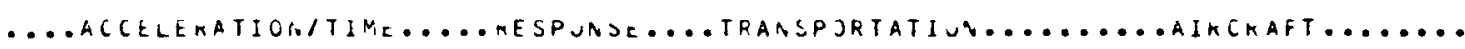

ULST LUAL FREGUEACY, AIKCKAFT FLIUHTS, LU-45KILGFETT, IV STCRM UTEET-JUZ

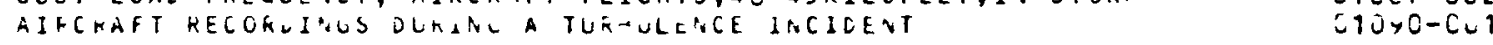

- . A A ACELLMATIGV/TIP SENERAL

$01801-020$

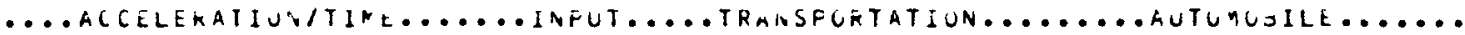

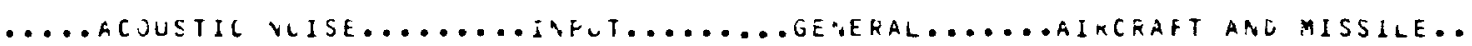

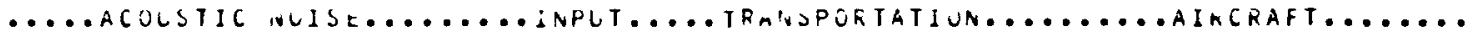

SOUAC PKESSUHE LEVEL VS ANGLE AI COL FEET,C1C4, ENGINES LP SOLVL PKESSUKE LEVEL US ANGLE AT E FEET,C1C4, D ENOINES UP UVEAALL SUUNO PAESSLRE LEVEL VS AVGLE, CIC4, VAKIUUS CONDITICA SCUIVL PKESSURE LEVEL, AVLKACE, CONPAFISCN UF G133A ANU C1L. COMPAKLSGN UF L11C,C1C4,C 13:A A.V RIL-A-ECOO SPL IV COCKFIT SCLAL TKESSUTE LEVEL,CLEFLL, CAOIV, CIEZ AIRCPAFT

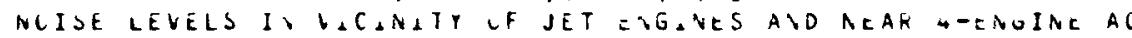
SCUAD FKESSURE LE UEL, UVLKALL, FUR DIX TURLUJET AI TERAFT, I JLFT SCURD PFESSLRE LEVEL,UVEKALL,FUR = TUKEUJET AITCKAFT, GJL FT

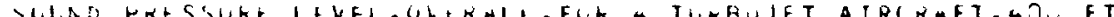
sise $13-\cos 1$ coter COE $14-201$ cus $15-\mathrm{Cu}$ Zu $016-5 C 1$ Cᄂ $17-5 u 1$ こした1ะ-こし1 Eve19-こL1 ue C C - ju 1 je $1-i+1$

SCLND PKEDSUKE LEVEL, UVEKALL, MAXIMUL, L USAF TLREUJEI A/C 


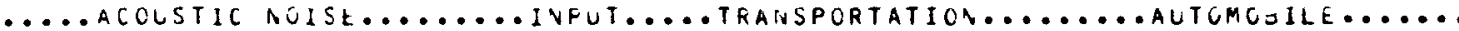

CONTHUL OF HIUHWAY RUISE

ACCUSTIL NOISE SQURCES. TKUCK

NUISL, TRANSHURTATIUN, SURFACE ANL AIK

COACEPTD FGK NUISE KEDLCTION IN TKACKED VEHICLES

$01134-043$

C1006-001

(16<4-001

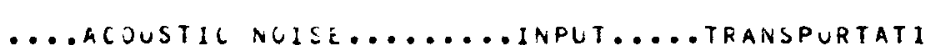

$\angle C>0-040$

ACCUSTIC NOISE SCURGES, TKAIN

$01539-005$

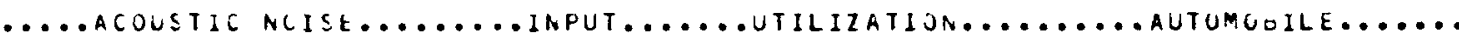

SURVEY THLCK NUISE LEVELS AND THE EFFECT OF REGULATION

$C 2 C<5-001$

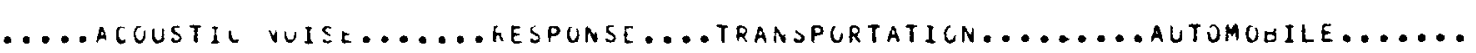

CCNCEPTS FOR NUISE HEDLCTION DU TKACKED VEHICLES

$02050-040$

. Atricsphekic cuntents........ INPUt.

$00078-001$

EAVIKONMENT COVOITIUNS FREVAILINO DURING TRANSIT

SALT SHKAY RAKIICLE, IN AIR, D, STRIELTION, CONCENTKATICN, SIZE

$00<74-001$

SAND ANL LLST-CHACTERISTICS

$01274-004$

.. atrusphenil cuntents...... input.........stufage............

AIKUCRNE FAKTICULATES

$01508-001$

.. atmuspheáll cuntents...... input.....trandportatiun.........

SARUIUUST IV HELICOPTEKIVSTCL A/C FLIGHTD

$01004-0 L 5$
$01009-04 C$

FMELLL CY U OCCLMEICE UF A IRCRAFT DCIAO FARAMETERS

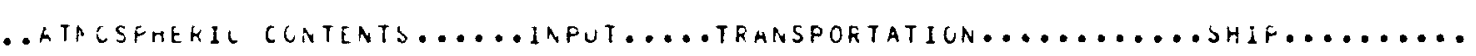

IEA SALT EROIKUNEET ON OLCK AT DEA

C1179-5Cc

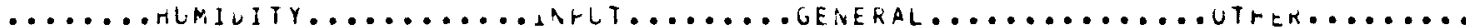

ENLIRUNAENT COHLIIILAS RRLVAILIEU DLRINO TRANSIT

MUNISITY, RELAT, VE, I? DESEKT, TKOFILS,AKCTIC ANO IA SHIPHOLOS

$\operatorname{CED} 78-5 \cup 1$

HUNDITY LXTREPES,PKOEAELE,FOK STORASE AOD TRAUSIT 
ENVIKGIMENT CUVEITIUNS FKEVALLING DURINU TRANSIT $\therefore C \leq \supset 7-2 C 1$ MAITFALL, AVERALE KATES UF Cuतso-0i 1

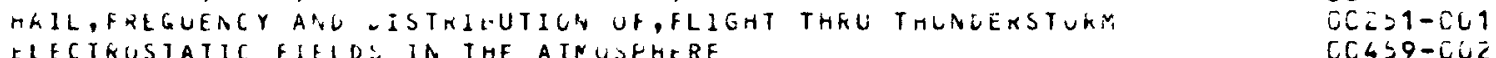
ELECTKUSTATIC FIELD: IN THE ATRUSFHERE

NAINFALL DIVTENSITY,FKEGUEVCY,U.S.,5J STATES,FLFTO RICO HAILSTOME HATKEMES

SLAZE ICE, IINL LOALS AT EAKTHS DURFACE

$01113-325$

$01513-001$

$1722-041$

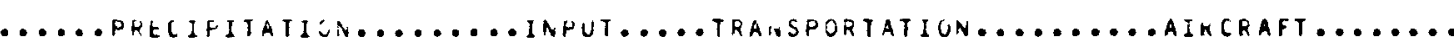

STATIC ELECTHICITY ACCUNULATION UR HELICOPTERS

CO4 $403-001$

FRE ULEP.CY OF OCCUREICE UF AIRCKAFT ICINO PARAMETEKS

$01009-002$

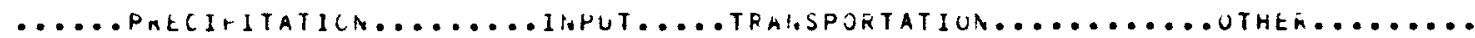

RAINFALL INTENSITY, DUKMTION, HAMAII

$01701-061$

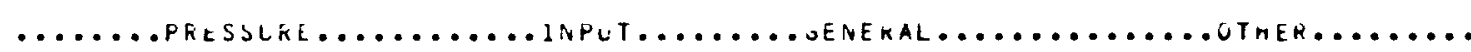

PRESOUKE, TYHICAL DAILY VS TIRE UF DAY

$00140-001$ BARURETKIC VALLES, MAXIMUN NUKMAL VAKIATIUN,UNITED STATES

$00143-001$

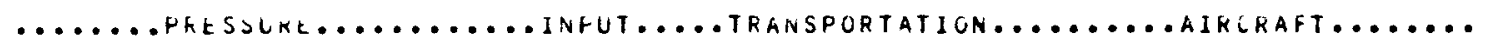

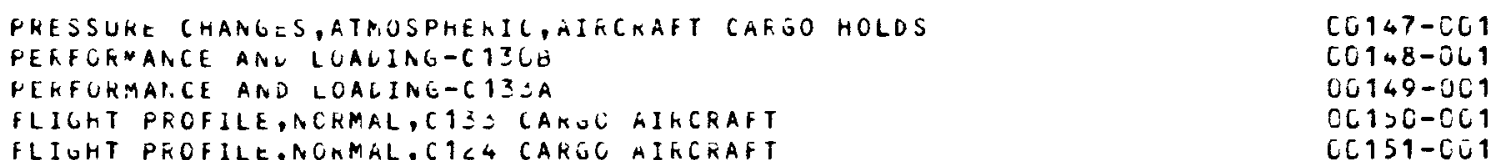

FLIUHT PROFILE, NORMAL, C $1 \angle 4$ CARUC AIFCRAFT

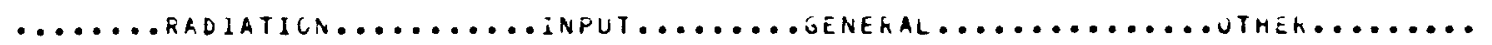

ENVIKONMEAT CONCITILNS FREVAILINO DURINO TRANSIT

IEUFERATURE CETREMESIOLFATICN GGF STORAUE, HANDLIVLOTRAMSPCRT

Eucis--2ut ELECTROSTATAC IILLOS IN THE ATMICSPHERE

STATIL ELECTKICITY-ELECTRICAL CHAKALTERISTICS OF ATMOSPHERE

$\operatorname{CScO-OC2}$ KACIATIUR. SULAR, TARTH S SURFALE APD ATMOSPHEME S EDUE

FADIATIUN DATA, SULAK

RADIATIUN, MEAN ORILY TUTAL, FLAT SURFACE,LOCATIONS,US/CANACA

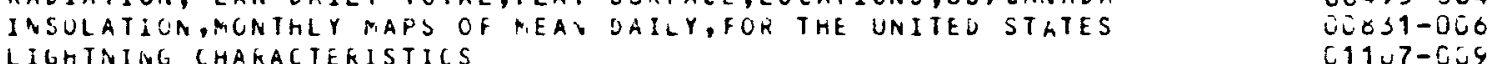

$C 0459-50<$
$0 C 4 C 0-002$

$00479-003$

$20480-001$

$0 C 445-304$

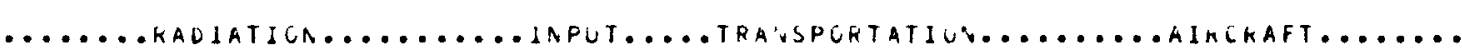


SHCCK FEÁSUREMENTS, UOCKSIUE/SHIFISUAFD HANOLING

PACKAGE LKUTFE CA LAFTH, WOUD ARU CONCRETE FLOORS

PACKAGES, TKEATMENT UF LUKIAG HARDLINE

SHOCK ZONES REUISTEKED IN CARCU JUR ANC HANDLING

SUNMARY, OYNAMIL ENVIRGAFENT, FORKLIFT TRUCKS

SHCCK, VILFATIUN, CÚO LU FOKKLIFT TKULK (SEE 1744)

SHCCK, INFUT, ZCCU LE FGRKLIFT TRUCK (SEE 1744)

SHCCK, INHUT, 4 CCOLO FURKLIFT TRUCK (SEE 1744)

SHOCK, VIOFATIUN, PLJU LE FGRKLIFT TRUCK (SEE 1744)

$00335-501$

$003: 7-001$

$\operatorname{cc} 343-001$

Cic $14-041$
$01744-031$

$01745-046$

C.1746-057

$01749-058$

$017>2-041$

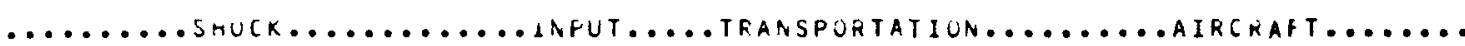

OYRAMIC EAVIKCANEAT,TUKEOJET CAKGU AIRCKAFT

SHOCK DATA, AIRLAAFTICARGOI

SHOCK ZONES FEUISTEKED DIN AIR CAKGO TESTS OURING FLIGHT CO3 $26-001$

SHUCK, CSA CAKUU FLCUK, ( $D E L 143$ Y)

$01444-042$

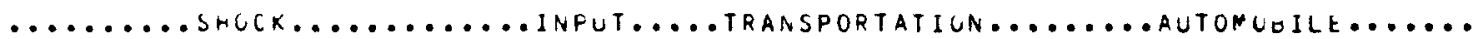

SHOCK SUMNAKY, TRUCK SHIMENT SO,UUD LE CASK SEE 2000

SHOCK SUMMAKY, TFLCK SMIPRENT 44, UOE LE CASK DEE $2 J 70$

TRUCK SHOCKIVIL LIV, LAFGE SHIPFIIVG CUNTAINER SEE ZUT1

SHCCK/VIE ENV LAKUE SHIFPING CCNTAINEK OY TRUCK PART

SHCLK/VIE ENV LARLE SHIFFINC CONTAINEK OY TRLCK PART

SHCLK DATA-TKULKS

SHCCKS FFUM ROAD IRKEGULAKITIES MIDD TOKEAR SNATCH

SHOCK ANE VIERATILA EAVIKCNMENT AO COMMERCIAL TRUCKS/TRAILER

SHOCK ANL VIERATILA ENVIKUNMENT D" COMMERCIAL TRUCKS

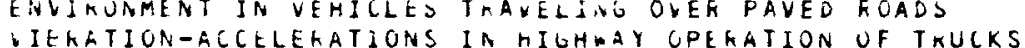

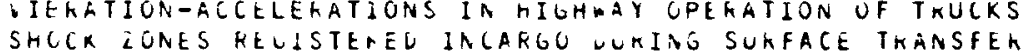

LYNAMIC EIVIROIMENT ON A HLAT ELD TRACTOK/TKAILEK (AEC/OUD)

FIAAL A\&ALYDIS, AELIUOD FLATSEU TRUCK TEST

VILKATION/SHOCK, C IIE TOR TKUCK

VIERATIUN/SHOCK AIR SUSPENSIOAT WAN

SHCCQ VVIEKATIUA SUMPARY,UAIUN CAREIUE TKUCK TEST

SHCCK DATA,UMICA CAKOIDE TKUCK TEST

ThALTOK/TKAILEK THANSPUKT, ? TYPES SUSPEASION SYSTEM

SHOCK FESFONSE. TRULK SHIHMENT $50,0 U$ LU CASK SEE CLOS

SHOCK RESPUNSE, TRUCK SHIFMEPT 44, CLI LD CASK SEE ZUCY

A $\angle 065-007$

A.2009-0U7

$A \bar{C} \cup 72-001$

AC104-010

Ac105-C10

$\cos 11-\cos$

$00314-001$

CC315-001

CL3 $17-0$ C 1

$\cos 70-001$

$00013-001$

c15is-008

C10 $13-041$

C1 $104-500$

C1 $677-500$

C1312-0C 7

C1314-013

C1988-035

C2u00-111

LCu>0-00t 


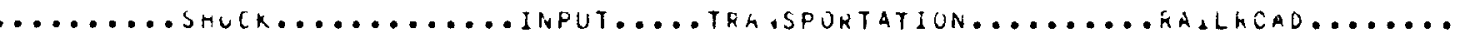

KAIL MUMF DNUCA CAITEKIA SUPMARY

KAILLAR SHCCKIVIE ENV, LARGE SHIPHING CUNTAINER

$A 1<v i-004$

LOXCAR SHUCK CKITLR A SUMA,AKY

ATHA CAM SHLCA CKITEKIA SLMNARY

SHCLA N SEKATION EAVIRONHENT SUMMGKY, RAIL TRAIUSFOKT

SHOCK SFECTIEA SUMNAKY, SIX-ATMX CAR TRAIIV

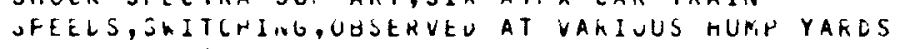

SPEEUS,SWITCHINC, CESERVATIOA UF, AT TLLIRUIS CENTKAL RK YARLS

SPEEOS, SWITCHIAU, UESERVEO

SHCCK, SWITCHING, RAIL, OCCUKANCE UF

SFEEU,SWITCHINO. DATA CCMPAEED WITH NEM DATA,NY LENTKAL KM

DPEED, HUMFING, FREGUENCY CF IMPACTS AT VAKIOUS

AAIL SWITCHIVU AMD HUMPING UPERATIONS

SHCCK DATA,RAILKOAD SWITCHING

SHCLK DATA, MAILKCAD SWITCHING

SHCCK DATA, KAILROAD SWITCHIAG

SHCGK DATA, EAIL

SHOCK VATA, KAIL/EGXCARI

SHOCKS IN IRALNS WHEN STORPING, STAKTING AND SWITCHING

SHOCA, RAILHOAD HUMPING

KAILKOAD SHITCHIAG TESI OF SPECIAL RAIL CAR ATMX $50 C$

KAIL HLMFIMU IEST, 5 LS SERIES ATMX CAR

IMFACT SFEED VS HCRIZONTAL TRAYEL OF STRUCK EMPTY CAR

VIERATIUN ENVIAONMENT PRODUCED LUKING RAIL TRANSFORT

SHCCK, RAIL HUMPING 3.4 TO 10 MFH, 3 DIRECTIONS

SHOCK KAIL RUMP SPELIAL DFAFT CAR, TO 1 CMIFH LONGITUDINAL

SHOCK SPECTRA, EOXCAK, LUNUITUDINAL AXIS

SHCCK SPECTRA, OCXCAK, VERTICAL AXIS

SHOCK SPECTKA, OOXCAR, LATERAL AXIS

SHOCK SPECTKA, SEFIES SCL ATNX CAR, LONCITUDINAL AXIS

SHOCK SPECTKA, SERIED SCU ATMX CAR: VERTICAL AXIS

SHCCK SPECTRA, SCC SERILD ATYX CAR, LATERAL AXIS

SHOCK SPELTRA, DEKIES CLC ATKX CAR, ALL AXES

SHOCK SPECTKA, HAKPEK CAK, SIX-ATHX CAK TKAIN

SHOCK SPELTKA, 1ST STRUCK CAK, SIA-ATMX CAK TRAIV

SHCCK SPECTKA, ZND STRUCK CAK, SIX-ATMX CAF TRAIN

SHOCK SPECTRA, SKD STRUCK LAR, SIX-ATMX CAK TRAIN

SHCCK SPECTRA, GTH STRUCK CAK, SIX-ATMX CAK TRAIN

SHOCK SPECTRA, $T$ TH STRUCK CAK, SIA-ATMX LAR TRAIN

SHOCK/VILKATION SUMMAKY, UNIUA CAKEIUE RAIL TEST

SHOCK LATA, URIUN CAKOILE MAIL TEST

SHITCHIIRG SMCCK, CUSHIUN UNDERFKAME LOXCAK

SPEECS, HAIL SWITCHING, DISTRIELTION

PEFFURMANCE FKEIGHT-CAF TEKS THKLUGR KOAU IESTING

TRAIN RUR-IN AND RUID-OLT FCRCE FIMLYSIS ENSCO REPORT

A $0079-510$

E $1 \dot{c} \cup 3-5 \tilde{L}$

$1<10-C G=$

E1 $1<0-011$

$1315-012$

- $3204-301$

पjटc4-jc

coces -50

$C 0<07-501$

COटट8-Ú 1

ᄃ0209-00

COC $90-C E 1$

$302 \times 3-502$

CUட४4-002

C0245-3uz

COLXE-001

$002 \times 7-001$

$00360-001$

$053 L 2-C 05$

$0 \mathrm{cos} 4-0 \mathrm{c}$

$00345-000$

C0349-301

C $0306-001$

01033-010

C1034-C04

$01<04-057$

C1205-je

$01206-055$

$01211-081$

C1212- 153

$01,13-018$

01214-025

C1216-070

$01<17-028$

C1 $118-015$

$01218-015$

C1द $20-015$

C12 $11-315$

C1515-007

$01317-033$

C1s<6-055

616 $18-001$

c) $145-005$

Cद $111-213$ 


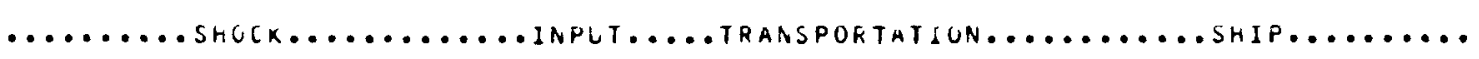

SHOCK SPECTRA, SHIP, TYPICAL

$00323-009$

ACCELERATION IN CARGO SHIPS DURIVU MANEUVERS

$01133-603$

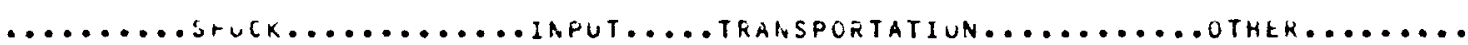

SUMMAKY, OYNAMAC ENVIRONMENT OF TKANSPORTATION - 1973

$01003-000$

TANSPURTATIUN OF EUROFEAIN SPACELAG COMPGNENTS

$02<10-050$

.......... SHUCK..........

SUMNARY, CYNAMIL ENVIROAMENT, FORKLIFT TRUCKS

SHCCK, VILKATION, COUO LE FORKLIFT TKUCK (SEE 1744)

SHOCK, RESPUISE, ZUOL LE F(RKLIFT TRUCK (SEE 1744)

SHOCK, FESPONSE, 4 CCCLO FUKKLIFT TRUCK (SEE 1744)

SHCCK, VIORATION, 7UJC LO FORKLIFT TRUCK (SEE 1744)

$01746-C 31$

$01745-040$

$01747-057$

$01750-058$

..........SHUCK...........KESPONSE....TRANSPORTATION.........AUTOMOUILE......

TKUCK SHOCK/VIU EAV, LARGE SHIPFIING CUNTAINER SEE 2071

SHOCK IVIO EDV LAFGE SHIPPING CGNTAINEF OY TRUCK PART

SHOCK/VIG ENV LAKGE SHIPPING CCNTAINER OY TRUCK PART

SHOCK/VIERATION SUMMARY, UNION CAROIDE TRUCK TEST

SHOCA DATA, UNICN CAKOIDE TRUCK TEST

A2072-0C1

$A 2164-C 10$

A2105-016

$01312-007$

$01314-013$

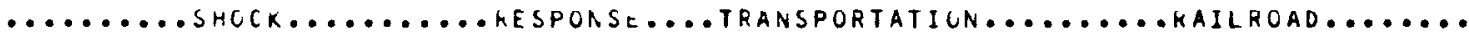

RAIL HUMF SHUCK CKITERIA SUTMARY

FAILCAR SHOCK/UIE EAV, LAKGE SHITHING CONTAINER

EOXCAR SHUCK CKITEKIA SUMHAKY

ATMA CAR SHUCK CKITLRIA SUMMARY

SHOCK/VILRATIO. EAVIROAMENT SUMFAKY, RALL TRANSHORT

SHOCR SPECTKA SUPMARY, SIX-ATYX CAK TRAIR

SHOCK SPECTKA, EOXCAK, LONOITUUITHAL AXIS

SHOCK SPECTKA, DOXCAK, VEKTICAL AXIS

SHOCK SHECTKA, EOXCAK, LATERAL AXIS

SHOLK SPECTRA, SEFIES SUL ATMX CAK, LONGITUDIAAL AXIS

SHOCK SPECTRA, SERIES SCU MTYX CAK, VERTILLL AXIS

SHCCK SPECTKA, JCC SERIES ATHX CAR, LATERAL AXIS

SHOCR SPECTKA, SERIES CLU ATMX CAR, ALL AXES

SHOCK SPECTKA, HAMMER CAK, SIX-ATAX CAR TKAIN

SHUCK DHECTKA, IST SIRUCK CAK,SIX-ATRX CAR TRAIN

SHOCK SPECTRA, LAD STRUCK LAR, SIX-ATMX CAR TRAIA

SHOCK SPECTRA, TKD STRUCK CAR, SIX -ATOX LAR TRAIN

SHOCK SPECTNA, TH STRUCK CAK, SIA-ATAX CAR TRAIN

SHCCK SRECTRA, TT STRUCK CAK, SIX-ATMX CAK TRAIN

SHOCX/VIEAATION SLMNAKY, UNION CAKSIOE RAIL TEST

SHOCK DATA, UAIOR CAROIUE KAIL TEST

A $1202-004$

A $2071-018$

61203- 002

E $1210-0 \cup 2$

B $13<0-011$

C1215-012

01204-057

$01205-004$

$01<06-05 y$

$01211-001$

$01012-053$
C1:13-01C

$01213-010$
C $1214-035$

$01216-090$

$01217-0<8$

$01<18-012$

C1219-005

$01<<0-015$

$01<<1-015$

$01315-C 07$ 


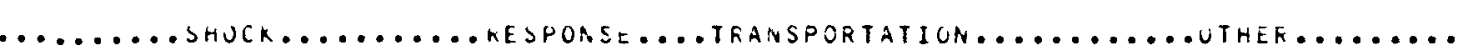

SHOCV + VIEKATION DATA OETAINEC FKON TPUCK + FAIL SHIPMENT CILU3-EOO

..........SHUCK..........

DYNAMIC FEASURERENT OF TRACK-INDULED RALLCAR HHEEL ACC C2016-006

$(1,1 F A(T)$

$(1 M F A C T)$

(IRISACT)

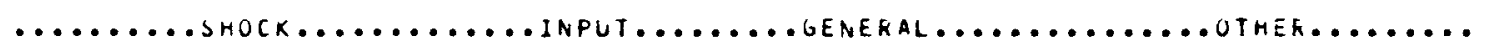

APFKUXIMATE DURATION OF SURE SHGKI ACCELERATION LOADS

$00734-001$

...........SHUCK............ INPUT

$01608-004$

MAXIYUM OKOPS, PACKAGES II, TRANSIT

\section{.........}

MAXIMUR DROPS, FACKAUES IN TKANSIT

$01608-004$

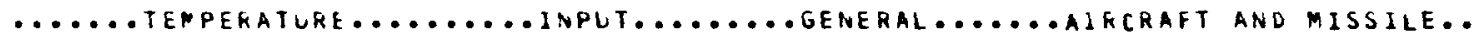

SPACE SHUTTLE UREITER VIEkOACOUSTICS

$02661-023$

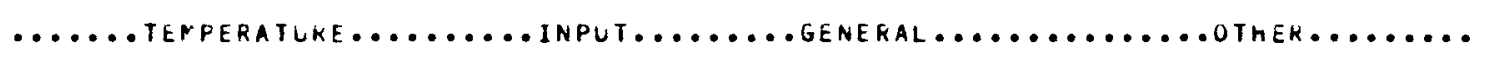

TEXFERATUKE REOINE FOR DESEET REUIONS

TEPPERATURES, $1^{\circ}-99^{\circ}$ DESIGN,FOR UNITEO STATES AND CANADA

$00006-002$

$00018-\operatorname{COC}$

ENVIKONMEAT CONDITICNS FREVAILING OURINO TRANSIT

CJ078-001

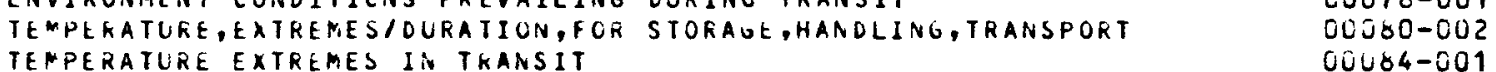

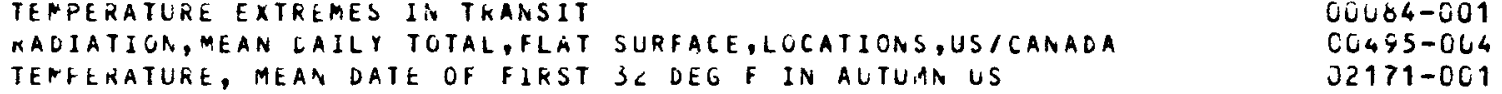

$\begin{array}{lll}\text { TERFERATURE, MEAN DATE OF FIRST } 3 \angle \text { DEG F IN ALTUAN US } & 32171-001 \\ \text { IEMPERATURE; MEAA OATE CF LAST } 32 \text { DEG F IN SPRINO US } & 02172-001\end{array}$

TEMPERATUNE. MEAN MONTHLY NO.UF DAY 32 F AND BELUW US

TEPFERATURE, MEAN LENGTH OF FKEEZE-FREE PERIOD-DAYS US C C 174-OC1

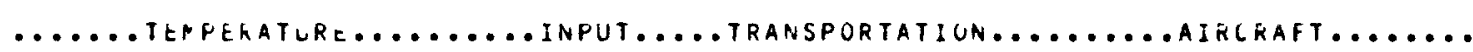

TEYPERATLKES, LARGO COMPGETMENT, C119C AIKCRAFT,ALASKA, INTER COUO9-201

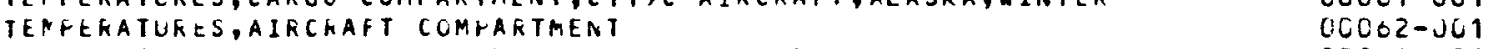
TEMPEKATURES, MAXIMUMIMINIMUM, IN AIR AND AIRCRAFT CARGO HOLOS CLOC4-UOI TENFERATURES, LUMPARTMEN $1,(141 \mathrm{~A}$

FRELUEACY OF OCCLREACE OF AIRCEAFT ICING PARAMETERS

$00066-0 C 1$

$01009-042$

TERPITIME DATA AIRCKAFT, TKUCK, TKA IN, ATMX

$01,08-004$ 


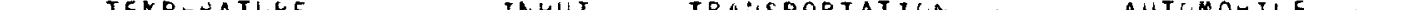

TERPERATUKE/TIME DATA,TKULK (DEE 12Z8)

$01349-004$ TENTERATUKE, TKUCK CAKLC, HILH AAU LCW

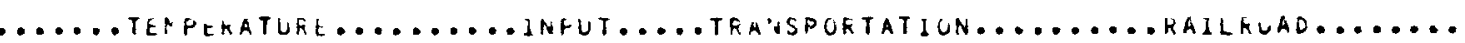

TEPPEKATUKE, I TTEFAAL AIK, RAILROAU EOXCAF IN DESERT

TEPFERATLKL TESTS, INTERNAL, KAILKOAD FREIGHT CARS "IMTER

OCu $56-001$

TE MFERATUREITIME OATA , RAIL

$00457-001$

C1310-004

TENPERATURE, HIGH, STANDING BUXCARS

$014 \cup 1-3<0$

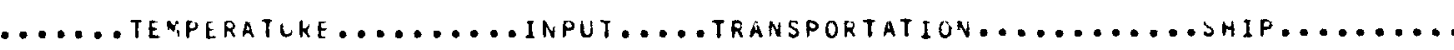

TEMPERATURES AND KELATIVE HLMIDITY,SHIP HULD CCU58-CU1

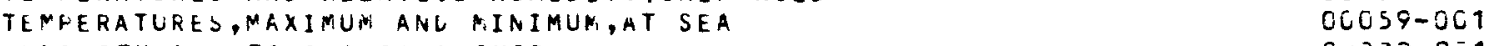

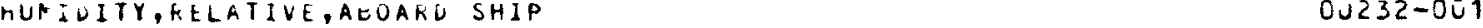
TEMPERATURE IN SHIPS HOLDS
$01202-003$

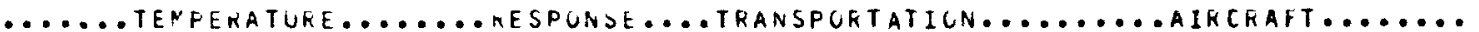

TENPITIME OATA AIKCKAFT, TKUCK, TRAIN,ATMX

$01208-344$

...... terteratuke........

TENFEKATURE/TIAE DATA,TRUCK (DEE 1208

TEFPERATURE, TKUCK CARGO, MIUH ANO LCW

$01309-004$

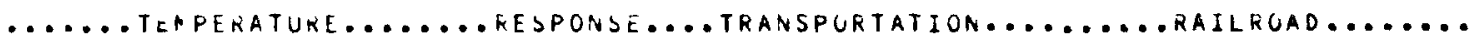

TEMPERATLREITIME UATA, RAIL ANO ATMX CARS (SEE $1<08$ )

01510-004

........

VIERATIUV UF HELICOFTERS (ISOLATIUN AND AOSOREERS)

$0<117-005$

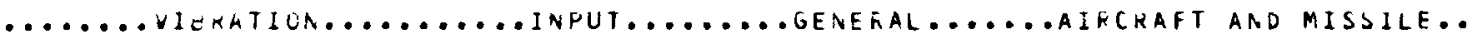

SPACE SHLTTLE UKEITLR VIUKOACULSTICS

$02001-023$

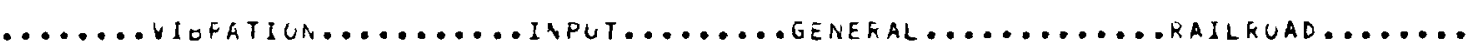

FAKANETRIC DTUUIES KR FRT CAR MATH MODEL HARMONIC ROLL C1900-005

MIDE WUALITY OH A CUNTAINER FLAT CAF E19C2-0UO

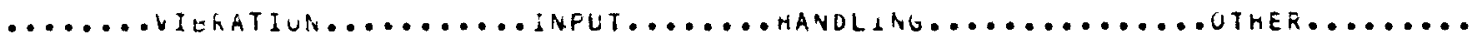

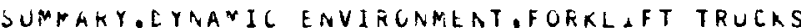

SHOCK, VIERATIUP., CCLS LG RORKLIFT TRUCK (SEE 1744)

$1744-331$

VIEKATIOR, INFUTIKESPONSE, 3 COU LO FORKLIFT (SEE 1744)

VIFKATIUR, INPUT/KESFONSE, $4 L O C$ LE FURKLIFT (SEE 1744)

c1745

SHOCK, VIOKATICN. 7LJC LE FLKKLIFT TRUCK (SEE 1744)

$01748-075$
$017>1-001$ 
WYAARIC EAVIRUNEEITT, TUREUJET CARUU MIRCKAFT

WIERATICA, MUST PRUBAGLE EXTKEME, PKOFCLLEK DKIVEN AIKCRAFT VIEKATION DATA,AIKCKAFY

VIEAATIOAS, MAXIMLLF LATERAL AND VERTICAL, HELICCPTEK FLOON VIEKATIUN,KL-1:5 FLCOR, VERTICAL, LATERAL, LONGITUDINAL

VIEKATIUN, FLCOK, FhOFELLLR, JET, HELICUPTER AIRCRAFT

VIEKATION, H-S7 HELILOPTER FLOUK, VERTICAL, LATEKAL FOKEIAFT VIEKATIUN, C-122 FLOUR, LUROITUOIUAL, VERTICAL,LATERAL AXES VIERATIUN, L-ISU FLOUK, VERTICAL,LATERAL,LUNGITUOINAL AXES

RESPUNSE OF TUROOJET/PISTUN EIVINE A/C TO RUNWAY ROUGHNESS VIEKATIOIN, AIR-KIUE VAN ANU $C-130, C-133$ AIRCRAFT

VIERATION NCISS AFT PASSEIVGER CUMPAKTMENT

VIERATIUP. SUMMARY, HELICOPTER, HHA E, UHT, CHO, CH4E, CH47

VIERATIUN, CARUO FLUOK, UH-1 HELICOFTER

VIEKATIUN, CARUU FLOUR, OH-E MELICUPTER

VIERATIOA, CARCU FLOUR, CH-40 HELICOPTER

VIERATIUN, CARGU FLOLR, CH-4T HELICUPTER

VIERATION. C1SUA,C1ZJY CAEGU FLUUE

VIFRATIUN C13U,CIS3 TAKE UFF AN. CRUISE

VIEFATIUN, CSA CAFGC FLOOR - SUMMARY - SEE 144 C TO 1444

VIERATIUN, CSA CARGU FLUOK, STANDARD VIURAN (SEE 1436)

VIENATIUA, CSA CARGU FLOUK, FOKMAT C VIORAN, (SEE 1437)

VIETATICA, CSA CAKUU FLOUK, PSD (IEE 14SY)

VIERATIUN, GSA (ARGC FLCOR, PSD, (SEE 143Y)

DYAAMIC ENVIRUNMENT, CIGI JET CAFUU AIRCEAFT - FIRST FLIGHT

LYAAMIC ENVIKUNMEAT, C141 JET LAKUU AIRCRAFT -SECOND FLIGHT

DYAAMIC EAVIRUNMENT, CSA JET CAFGC AIRCRAFT

VIERATION OF A LARGE TRANSPCRT HELICOPTER (CH-S3A)
A 15:3-0uz

$504 \angle C-201$

$064<4-301$

$0 \mathrm{C}+33-301$

Cue 46-UL1

โuc47-0̃ 1

Cuc40-ju1

30 E $51-0 C 1$

$300>2-3 x 1$

ECY10-jú

$011+8-608$

$01218-510$

C $13<1-009$

$012<2-007$

$013<3-307$

(1) $<4-043$

(1) $134-C 43$

$13<5-[31$

$01356-001$
$01557-001$

(1438-629

$01440-0.05$

C1441-030

$01442-088$

C1443-095

$01534-024$

$01534-024$

$01535-032$

$01536-080$
$02074-005$

........

VAIL SUMAARY,TAUCK SHIFMENT OF 50,000 LO CASK SEE 2603 VAIL SUMMARY, TRLCK SHIFMENT 44, UOU LE CASK SEE 2067

TRUCK SHCCKIVIO EAV, LAFGE SHIFFIAG CONTAINER SEE 2071 TRUCK SHCCKIVID ENV, LARGE SHIFFING CONTAINER SEE 2071 SHOCKIVIE EAV LAKGE SHIPPING COATAINER EY TRUCK PART 2 VIGRATIUN, PKGGAELE MAXIMUR, TRUCK, TRAILEF, TRACKEU VEHICLE VIERATION SUMMAFY, DTANOAKD FEEIUHT TRAILER REOLÜ28 VIEMATIGA-ACCELEFATIONS IN TIGHWAY OPERATION OF TRUCKS VIERATIUN IA RUAL VEHICLES

VIEKATIUA, MAXIMUY, ON TRUCKBED, VARIETY OF FOAD OPEKATIONS VIERATION, MAXIMUM ACCELEKATION IN CARGO TRUCK

VIEKATICN DATA, TYPICAL, VATV TRAILEK, AOFMAL SUSPENJION VIOKATIUA, FLAT-DED TRACTOK-TRAILEK

VIEKATION, TRUCK FLOUR, EMPTY AND FULLY LUAOEO

OYNAMIC EAVIKOAMENT ON A FLAT GLD TRACTUR/TRAILER (AEC IOLD)

FINAL ANALYSIS, AECIUOU FLATEED TKUCK TEST

VIGRATIUPA EMVIROPMENT, AEC VAN WITH TRAASIT RIG SHIPPIIVG CONT

VIFAATIUN/SHUCK, $21 / 2$ TON TEUCK

A20204-004

A2CO8-0C4

$A<072-001$

AL104-010

AC105-016

GCSO7-50

$69548-C 69$

$00370-001$

$\cos 34-001$

[0₹77-501

CC $379-001$

$003 \times 7-001$

$00401-001$

$60 \times 49-001$

c10us- C08

01 uc $3-041$

C1:45-001

C1204-068 
........ VI

VIERATIUN/SHCCK AIR SUSPEASION VAN

SHCCKIVIERATION SUMPAKY, UNION CAFEIDE TKLCK TEST

$01277-000$

VIVFATIOR GATA, UITUN CAKEICE TKUCK TS

$01312-007$

VIERATIUA, TKUCK, TANUEM AXLE, AIR DAG SUSPENSION

VIEKATION IN CUMMON MOTOF CARRIEKS

TRACTOK/TRAILEK TRAISSPCKT, Z TYPES SUSPENSIUN SYSTEM

$01313-045$

$01301-001$

$01742-007$

$01508-605$

VIERATION, SPECTKAL DENSITY, STANUAKD FREIGHT TKAILEK

$14 \times 7-235$

COACEPTS FUA NUISE AEDUCTICN IN THACKED VEHICLES

$0<550-040$

$0<052-007$

FSO STANDAKD FREILHT TKAILEK KE-KEDUCTION ALSO SEE1SY?

$02003-113$

VIPKANIPSO, TRUCK SHIPREAT UF 50, LOU LE CASK SEE 2064

Cथि $567-009$

........vigkatiun........... infut.....transportation.........kallkoad.......

KAILLAF SHUCK/VIE EAV, LAKGE SHIPHING CONTAINER

SHOCK/VIOKATION ENVIRONFENT SUMMARY, RAIL TRANSPORT

$\begin{array}{ll}\text { VAIL SUMFARY, MTKX KAIL CAR TEST FEOU } 403 \text { SEE EDO } 2079 & 82080-019 \\ \text { VIERATIUN,FAIL TRANSPORT, SUMMAKY UF DATA ON } & 00355-601\end{array}$

$A<679-018$

$81320-011$

$\begin{array}{ll}\text { VAIL SUMFARY, ATRX KAIL CAR TEST FCOU } 03 \text { SEE EOD } 2079 & 82020-019 \\ \text { VIERATIUN,FAIL TRANSPORT, SUMMAKY UF DATA ON } & 00355-601\end{array}$

VIBRATIOA DATA, KAIL

VIEKATIUN TESTS, FAILROAD, SPEED LUMPH

VILRATIUR TESTS, KAILROAO, SPEEU $73 \mathrm{MPH}$

VIERATION TESTS, RAILROAD, SPEED EUIAPH

VIBKATIONS IN KAILRGAD FREIGHT CAFS AT SPEEDS FROM 31-73MPH

$00357-001$

$\operatorname{CO} 3>8-061$

$00359-001$

$00300-001$

$00302-001$

VIERATIOAS MIEAOUREO ON ATMX SUL CAR AT SPEEOS 3U-7OMPH

$00363-001$

VIThATIUA ENVIKUTMEAT PROLUCEC DURING RAIL TRANSPORT

$00360-001$

cos $360-001$

$00850-001$

VIEKATIUN, RAILROAD, JOFT SPRUAG CAK, VERTICAL AND LATERAL

VIKKATION, FAILKOAD ENVELOPES DIRECTIONAL COMPCSITES

SHOCK/VIEKATIUO SUMMAKY, UNIUN CAROIUE RAIL TEST

C10S1-001

VIEKATION DRTA,UNION CAREIDE KAIL TEST

$01032-001$

$01316-005$

$01328-084$

VIERATION, KAIL EOX CAR WIEMPTY, HALF AND FULL LUAD

$02079-031$

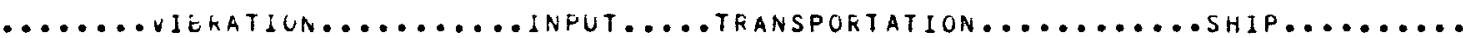

VIEKATIUA, WUST EXTREME HKGBABLE, EXPECTEO IN SEA TKANSPORT

VIBEATION FEA SURENENTS UN SHIPELAKD DURING TRANSPURT

VIFRATIUA UATA, SHIPS

VIEKATIJA DATA, SHIPS IN STRAIGHT KUNS AT VARIOUS SPEEDS

VIEKATIUN, SHIFDUARD, NOKMAL STEAMING AND HARL TUNAS

VIERATLUR DATA, SHIPS, HIGH SPEEL ANEUVEK ANO LRASH EACK

VIBKATIUNS, SHIREUARU, FRLM VAKIOUS SOURCES

VIERATJUAS, MEKCHAWT SHIF SS WULVERINE STATE

EOLL4-UC

CCL $202-001$

$00465-001$

Cᄂ4 $47-001$

$\cos 08-001$

$00404-601$

$00415-019$

$00412-001$

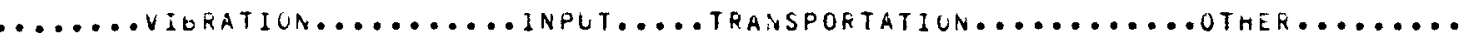

SUMMARY, OYIVAMIC ENVIRUNAENT UF TKANSPORTATION - 1573

A $12>24-018$ VIBRATIOR. SUMMARIES GF EDE1354 IN PDD FUKMATS

$121354-360$

VIEKATIUN-MAXIFUN ALCELEKATIONS FUR VARIOUS VEHICLES

C०308-SC 


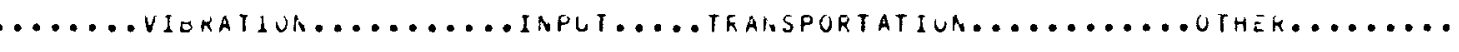

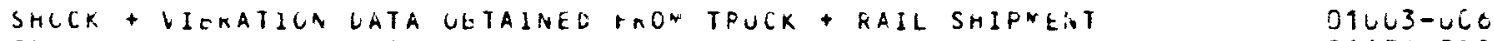
TRANDPURTAIION VIUKATICA EAVIRONMLNTS $\quad$ C1176-CUZ

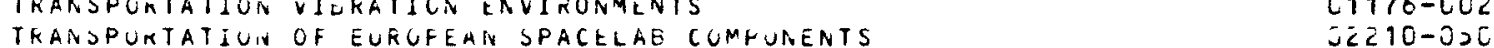

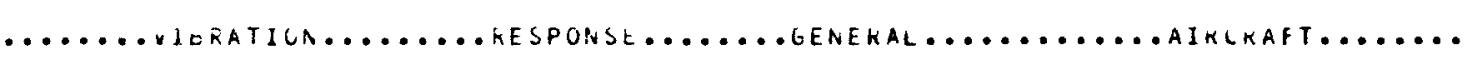

WIEKATIUH OF HELICOPTEKS (ISULATSUN AND AOSOREEKS) CZ117-0L5

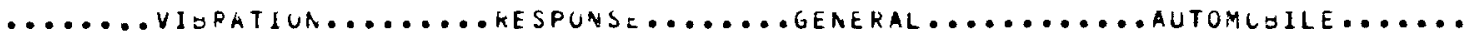

STUOY OF FASSENGEE TESPONSE TO IUEAL ANO REAL VEH VIb C1941-OC1

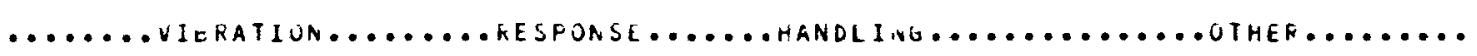

SUMPAKY, CYNAMIC EAVIRONMENT, FOKKLIFT TRUCKS

SHOCK, VIERATIUV, COCU LO FOKKLIPT TKUCK (SEE 1744)

VIFKATION, INPUT/RESPONSE, Z UOJ LO FORKLIFT (SEE 1746)

$01744-031$

VIEKATIUN, INPLT/KESFONSE, 4 COO LE FORKLIFT (SEE 1744)

$01745-046$

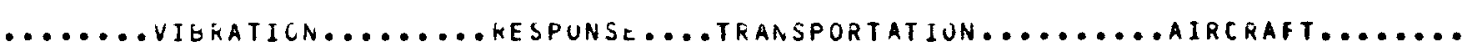

VIERATIOR NC13 2 AFT PASSENGER COMPAKTMENT

$01318-010$

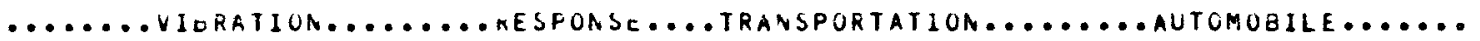

TKUCK SHOCKIVIL EAV, LARGE SHIFPING CONTAINER SEE 2071

SHOCK /VIE ENV LAFGE SHIPPING CONTAINEK OY TRUCK PART

SHOCK/VIP ENV LAFGE SHIPFINU CONIAINER OY TRUCK PART 2

A $2 \cup 72-0 C 1$ VIERATIUA EIVIRUNMENT,AEC VAN WTTH TRANSIT KIIG SHIPPING CONT SHOCK IVIERATIOA SLMMARY, UNICN CAKEIDE TKUCK TEST

VIEKATIUN DATA, UNIUN CAREIUE TRUCK TEST

CONGEPTS FOK NOISE KEDLCTION IN TKACKED VEHICLES

$01312-007$

C1313-045

$0<\cup 50-040$

....... vivienatich......... kesponse.... transpoftation.........

KAILCAK SHOCK/VIE EAV, LAFGE SHIPFING CONTAINER

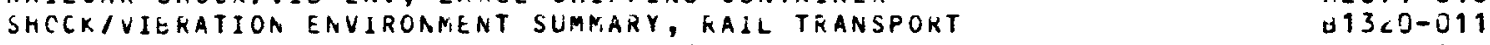
VAIL SUMMAGY, ATMA KAIL CAR TEST KEJO4O3 SEE EDE $2075 \quad$ GCOO0-019 SHOCK/VIEKATIUN SUMMARY,UNIUN CAR̃OIDE RAIL TEST O1315-DUT VIEKATION DATA, UNIOA CAKEIDE KAIL TEST

VIEKATION, KAIL BCX CAK WIEMPTY, HALF AND FULL LOAD

$01316-005$

$013<8-004$

VIFRAN/PSD, ATPA KALL CAR TEST ROCOAC3

$52079-031$

........vieratica..........

SHCCK + VIURATIUN DATA CBTAINED FROM TRUCK + RAIL SHIPMENT

$01003-000$ 


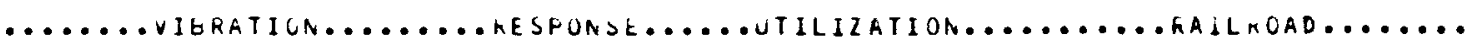

DYAAMIL MEASLIEAENT OF TKACK-INDUCED RAILCAR WHEEL ACC

¿̇ंบ16-Cuo

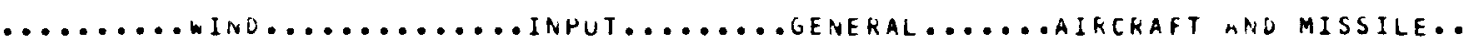

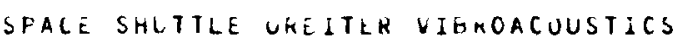

$0<001-023$

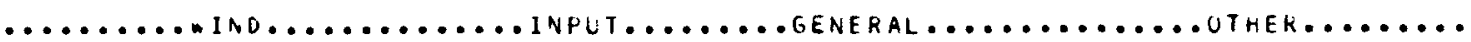

EAVIFONMENT CONDITICNS PEEVAILING DURINO TRANSIT

WINO VELOCITIES, WORLDWIUE, AND DISTRIBUTION IN TEMPERATE ZONE GC258-OCL1

- IRD SPEED DISTRIGUTION IV THE UNITED STATES

C1545-005

GLAZE ICE, WIAD LOAUS AT EAKTHS SURFACE

$01722-001$

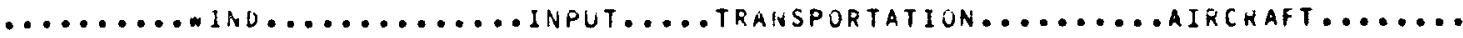

HATIGUE DARAGE PARAMETERS, TKAMSPUKT AIRChaFt

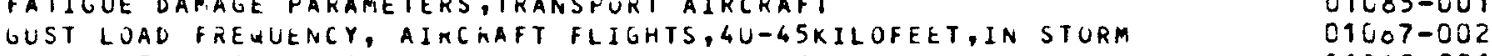

$01085-001$

AIRCKAFT RELORUINGS DURING A TUKOULENCE INCIDENT

$01050-001$

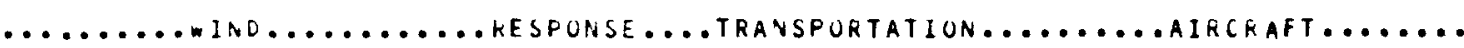

SUST LUAC FREGUENCY, AIKCKAFT FLIGHTS, 4U-45KILOFEET, IN STORM

$01007-0032$

AIKCKAFT KECUKLINGS DURING A TUKOULENCE INCIDENT

c1050-001

..........mind............ Input........ general............

WIND AND MAVE MEIGHTS - TKOPICAL STCRMS

C1036-0C2 
• 
Abnormal Environments 


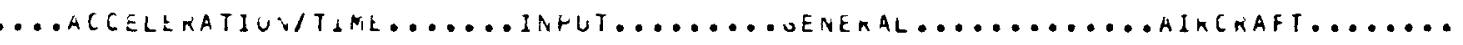

ACCILENT KATES, CIVAL AIRLRAFT 1YC4-1474 CIVIL AIRLRAFT ACCILEVT TYPLS, 1>7C - 1\$74 AIECKAFT ACLILEKT REPCKIS - NTSE - TY7C

AIRCKAFT ACCIUENTS, TYFE, RATE, INTSE IS?

AIFCRAFT ACCIOELTS, TYFE, RATE, NTSE TST

ATSE TY74 KE HJRT TO COUNRESS. AIMCRAFT

SLAMAKY US CIVIL AVIATIGR ACCADEVT STATISTICS 1YO5-75

AIKCKAFT ACCIDCNTS INVGLVING FIKCS $1903-1974$ ANALYSIS

ACCILENT RATES, CIVIL AIRLRAFT $9 \times 74$

LCPFARIJCA 1574 CIVIL A/C ACCIDERT KATES TO 1904-73

ACCDUEAT KATES, CIVIL AIRLEAFT, 1>63-197:

ACCIOENT FATES CIVIL AINCEAFT $9740-197$

ACCILENT KAIES SY MAKE APL FOUEL 1973-197E SEE DE $2 C 0$,

ACCIDENT KATES US GENEKAL AVIATIUN 1907 TO 1977

ACCIDENT KATES CIVIL AIKCFAFT IYC7-1977

ACCIDENT KATES EY MAKE AND MODEL $940 C-1$ Y 07

AIFCKAFT ACCIDENTS INVGLVIAUO FIKES 1905-1974 SPECLAL

ANALYSIS LS NAYY MAJOK AIKCKAFI ACCIDENTS 147Z-1Y74

AIRCKAFT CCLLISICAS WITH FLYING ILLLIFE (OAT HAZARU)

$41234-006$

$41673-265$

$017<4-3 \cup \bar{C}$

$1750-0,5$

$617327-310$

$617>7-310$

C14 $144-604$

$015<3-007$

$01940-002$

$01742-004$

$01943-007$

$01 \times 29-067$

Cदcu5-06

C<ट02-ju 1

C<U OL2-

$021<8-001$

$021<9-006$
$62134-C L 3$

02135-031

62151-053

$021<7-064$

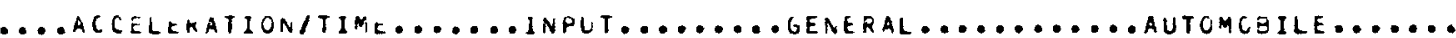

NTSO 1 Y 74 KEFOKT TO CUINORESS, AUTUMUBILE

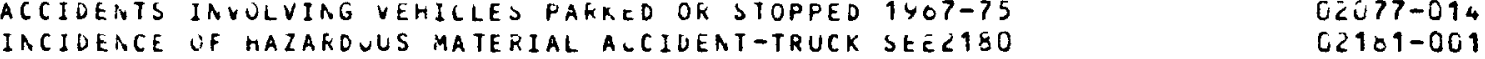

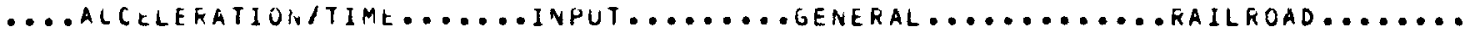

RAIL ACCIOEVT SPEED STATISTICS

FAIL ACCIDEMT STATISIICS, 1 Y6S

KAIL ACCIDEITT STATISTICS, 1471

NTSE 1Y74 KEFUKT TO CCNGKESS, KAIL

TRAIIN ACCIUENT FATES, TYE5-1974

A SAFETYICOST DTLUY SHIFPING SPEIT FUEL SPECIAL TRAIN

KAIL-HIGTWAY OKACE CROSSING ACCIDEATS 1965-1474

OFOKEN NAILS, MAJUR CAUSE OF TRAIIN ACCIDENTS $170<-147$

INCIDENCE OF HALARDUUS KATERIAL ALCIDEAT-RAIL SEEZ12J

$310<0-002$

$31606-00<$

$01007-002$

$01906-001$

C15 $120-004$

C2บบ9-017

$0<113-003$

$02137-010$

Cं. $102-001$

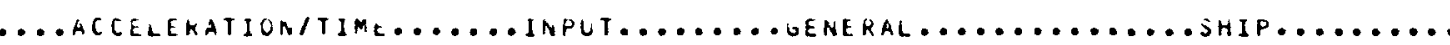

SURMARY FRT SHIFS AND FKT BAFUE ALCIDENTS 1905 - 1974 SLNMANY US TAMKSHIPS AND TNK BAKGE ACCIDENTS 1903-1975 IACIUEACE UF HAZARDUUS MATERIAL ACCIDENT-SHIP

$01>05-005$

$01542-005$

CONPEKICAL VESDELS THAT CARKY FKT IN ACLIDENTS 1903-74

$02180-C 41$

PUELIC CAREU VESSEL INVULVEL IN ALCIDEATS 1963-7.

FRE1GHT EAKUE ACCIDENTS REPORTED 1405-74

US INSFECTED CARCO FRT VESSELS ALCIUENTS FEFORTEDOZ-74

$\checkmark \dot{C} \cup 5-0<\bar{C}$

C2 $2 \cup 0-0<0$

$C<\angle \cup 7-143$

i2 $268-173$ 


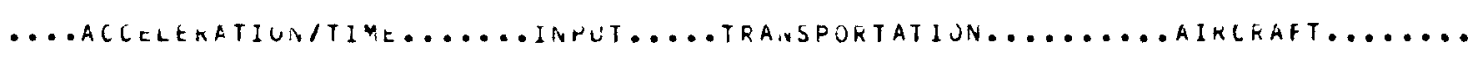

IACILENGE UNCUNTAINCD FUTLR EUKSTS LOKN AVIATIUN CZ-76 GC14C-CJE

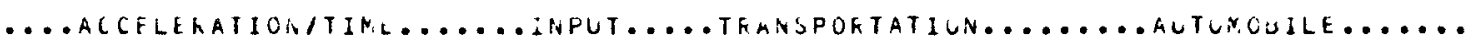

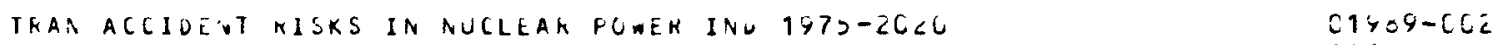
ARALYSIS ANO SUPYARY, ACCIOEVT IUVESTIGATIONS TY7Z-7O CIC144-EUY

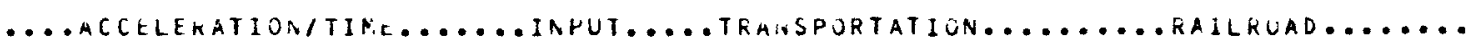

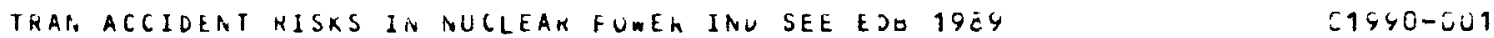

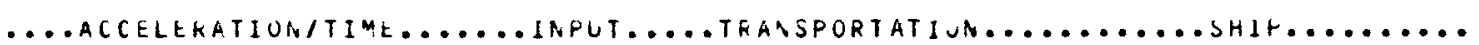

TRAN ACCIDENT KISKS IN NUCLEAR POAER IND SEE EDO 19C9

$019 \times 1-001$

(EARTHQUAKE)

(IPPACT)

(IMPACT)

$(1 M F A C T)$

...ACCELERATION/TIME...... INPUT

EAKTHUUAKE, STKONG MOTICN - ACCELUGKAMS G VS TIME

EARTMQUAKE, STRURG MOTION - ACCELUGRAMS G VS TIML

EARTHEUAKE - FUCK MOTIUN ACCELUGRAMS G VS TIME, DISTANCE

STRCNG MOTIUN EAKTHGUAKE ACCELOUKANS - PAKT F

STRCNO MOTION EARTHEUAKE ACCELCURAMS - PART G

STRUNO MCTICI EARTHLUAKE ACCELOURAMS - FART H

STRONG MUTION EAKTHLUAKE ACCELUCRAMS- VUL I,PT I, DATA

STRCNG MCTIUN EARTHEUAKE ACCELUGKAMS- VUL I,PT J, DATA

DTRONG MOTIUN EAKTHGUAKE ACCELOGKAMS- VUL I,PT K, DATA

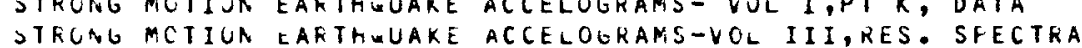

DIRCAG MCTIUN EARTHUUAKE ACCELOGRAMS-VOL III,RES. SFECTKA
STRUNG MITION EARTHWUAKE ACCELOGKAMS-VOL IV,FOURIEK SPECTRA

STKUNG MOTION EARTHWUAKE ACCELGGKAMS-VOL IV, FOURIEK
EARTHUUAKE, ANCHOKAUE, 1SOLC ENGIVEERING INFOKMATION

EARTHGUAKE ACCELOGRAMS-DIGITIZED AND PLUTTED DATA

EARTHGUAKE ACCELOGKAMS-FESPONSE SPECTRA

EARTHUUAKE ACCELOGRAMS-KESPONSE SPECTRA

EARTHQUAKE ACLELOGRAMD-FQURIER SPECTRA

$00448-0 C_{4}$

$015<6-004$

$015 \div 7-004$

C15 8 -0u1

C15 88-0u1
$01570-0 i 4$

$01570-014$
$01571-004$

$01572-004$

$01600-044$

$01601-004$

$01+42-394$

$01+\cup 2-304$

C1603-003
C16u4-00

C1001-0C

01 5 2-002

$01053-004$

$01<54-003$

$01055-00 s$

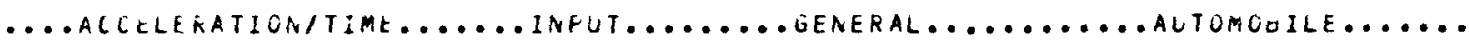

TRUCK ACCILENT SPEELS A - SUMMARY

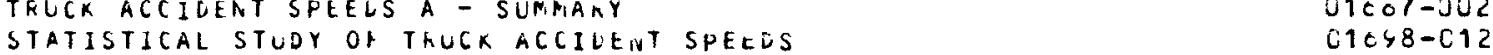

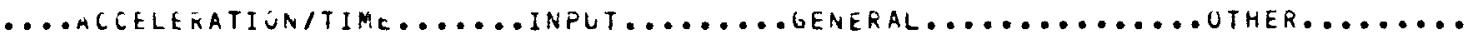

APPROXIMATE DUIATION OF SUME SHORT ACCELERATION LOADS

¿0734-001

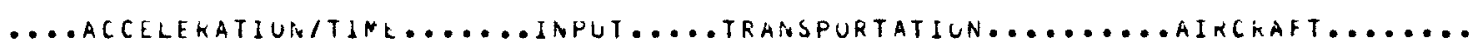

ANALYSIS UF DIRECIICIN UF LOADS IN AIRCEAFT ACCIUENTS

ACCELEKATION OF FLOUR-AIRCRAFT LFASHES

ACCELEKATION, AIRCKATT CRASH-VARIUUS ANELLS OF IMPACT

Ci>s $1-0 C 1$

$50752-\operatorname{cit}$

$00753-060$ 
TRUCK ACCIDCNT EAVIRONMENT ANALYSIS TAC - STUOY

ACCELEKATIONS, AUTOMUEILE CRASH (HEAD-UN)

DECELERATION PATTERIVS FOR AUTOMOEILE HEAD-ON COLLISIONS

IRUCK ACCIDENTS, NHTSA, FINAL REPORT

TRUCK ACCIDENTS, NHTSA, VOLO,NO.4

TRUCK ACCIDENTS, AHTSA, VULO, NO.5

TRUCK ACCIDEATS, AHTSA, VOLO, NO.O

TRUCK ACCIDENTS, NHTSA, VOLE,NO.7

TEUCK ACCIDENTS, AHTSA, VOLO,NO.:

TRUCK ACCIDENTS, AHTSA, VOLC, NO.

CAR ACCIDENT, NHTSA,VOL.O, NO.11

TRUCK ACCIDENT, NHTSA, VOL.6, NO.1

TRUCK ACCIDENT, NHTSA, VOL.O, NO.1C

TRUCK/PICKUP TRUCK ICAMPER AND TKAILER COLLISION

AUTO COLLISIGN WIANC COLLAPSE OF GRIDGE, SILOAM, NC

IRUCK ACCIDEAT, EXPLOSIUNIFIRE, EAGLE PASS, TEXAS

SCHOOLBUS/AUTO COLLISION/ROLLOVER, ASHLAND, OREGON

COLLISION OF TKUCK AND EUS, NEAK HAPILTUN, GA JUN 1975

C1944-002

$00737-001$

$00738-001$

$01875-019$

$01376-003$

$01877-004$

$01878-000$

$01879-006$

C1880-011

$01 \measuredangle 89-014$

C1848-005

$01549-005$

$01800-004$

$01901-015$

$01952-014$

$01953-011$

$01954-017$

COLLISION OF TKUCK AND BUS, NEAK HAPIILTON, GA JUN 1975

$019>5-009$

$01956-015$

$01957-006$

COLLISION OF TRUCK AND CAF, LOVINGTCN, NM 3 JAN 1976

CAR COLLISION, FRONT-TO-FKONT IRPACT TEST AT 60 MHH

TRUCK TRACTUR-TRAILER/GKOUNO/ROLLOVER VOL 7, NO 1

TRUCK TRACTUF-SPOFTS VAN IMFACT VGL 7 , NC 1

TRUCK CAR ACCIDENT, GALTIMORE, MD, ON INTERSTATE

$01902-004$

$01903-005$

$01904-000$

TEUCK CAF ACCIDENT, REAK END UNLEK-RIDF IMPACT, VOL 7

$01905-000$

GASOLINE TRUCK ACCIDEAT, SEATTLE, MA 4 DEC 1975

$01906-001$

TKACTOR TRALLE/CAR/SCHOOL UUS, REAK END, ROLLOVER

C1907-005 
CAR OUS ACCIDEAT, OVERLANU FAKK, RANSAS 7 CCT 1975 STATIUIN MAUUN/TKALN ACGIDENT, OALTIPORE, MD DEC 1475 TKALTOR TKAILEK, MEAK END AIVD KCLLOVEK AY I JUL 1975 IRUCK ACCIDEI,T, TIKE OLEL OUT, I"FACT, VY 21 JUN 1975 STATION WALUA./SCHOOL BLS, HEAO UA, MAMBUFG,NY SEP 1475 TKLCK/SCHGUL OUSITRAIA ACCIUENT ARKANSAS 11 SEP 1975

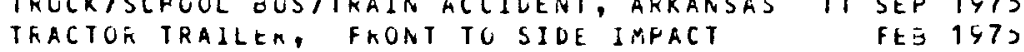
THLCK CAK ALCIDEST INTEKSECION, SALTIMONE,MD FES 1Y75 THLCK CAK ALCIDEST INTEKSECION, UALTIMOKE,MD FES 1475
CAR/PULE/TKAIN SIDESWIPE IMFACT, LUS ANELES 25 SEP 1S75 TRUCK CAK ACCIDENT, HEAR END, SAV DIEUO FREEWAY JAY 1 STO UNDERIDE OVERIDE INTERVEHICULAL COLLISIUVS

TRUCK ACCIDEAT, THI-STATE RIG, GILA JEND AZ

TRUCK ACCIDENT, COLLISION LEFT KUADWAY AASHINGTON DC

TRUCK/SCHUCL BUS COLLISIOA LAKE FLACID TL

TRLCK IVAMIALTU ACCIUENT LEXINGTGM VA

LUNOER TFUCK ACCIDEUT-CAFGO SHIFT HAKTFURD O DEC 1975 TRUCK TTKAIA CROSSING ACCICENT NY ROUTE 10 10 MOV 1473 IRUCK, FRONT COKNEK INPALT, CONCOKO, NY 27 NUV 1973 TKULK WHEEL/CAK - TGP IMPACT VY DTATE 26 NOV 1973 CAR/TRACTUR-THAILER UNUCKKILE CULLISION 13 DEC 1474 TKACTOK-TKAILEK CAMPING ACCIDENT, HARDIN, KY JUN 1475 MOTOK HUME OKAN-OFF ROADAAY, IMPALI,FIRE, EXPLOSIUN 1 1Y75 TPACTOROTFAILEKUCAR SIDESWIFE FUHAL XY TRACTOR CARAILEKICAR SIOESWIFE COAUT HIGHWAY 17 JUN TRUCK CAR ACCIDENT PACIFIC COAST HIGHWAY 17 JUN 1 Y75 WHITE FREIGHTLINEK ACCIOENT TOUE TKAILER TRUCK TKAILEK ROLLOVER, POOK GRAKES, VERN. CA JUN 1975 TRUCK CAR ACCIDENT, LANE LHANGE, LOS ANOELES JUN 1975 TANKTRUCK ACCICENT, HELIUN, FUEL LEAKAGE JUL 147ל JACKAIFING TRAILER FORCED TKACTCR INTO GRIDGE JUL 1975 TRUCK TKAILER ACCIDENT, CARCO SHIFT, ROLLOVER AUG 1975 TRUCK ACCIDENT, PROYANE, OVERTURY AND FIFE 9 MAR 1572 CARITKAIN, URADE CRCSSIAG, DES MOINES, IUWA DEE DQ 2085 CARACTOR TKAILEK COLLILED WITH CAK GAXTER, CA II MAR 77 TRACTOR TKA ILEK COLLIUED WTH CAK GAXTER, CA II MAR TRACTOR/CANGO TAAK IRAILEK ACCIUENT, CHEKRY HILL, NJ
TANKTRUCK/RAIL GFADE CKUSSING ACCIOENT SEE DO CUSS SCHOOL DUS TRAIN ORADE CRUSSIAG ACCIDENT SEE DB COYE TRACTOK-SEMITRAILER COLLISIOV MULTIPLE VEHICLES ALG 70 MINI MOTOK HUME/CAR ACCIOENT MCALESTER, OK 14 JUL 77 TRUCK ACCIDENT, PENETRATEL ERILGE RAIL KELEASED HM CG IAAKTRUCK FAIL CFUSSING ACCIDEAT SEE DEC118 10 MAR 75 TRUCK ACCIDENT, GASULINE FIRE, DEATTYVILLE KY US/C4/77 TRLCK RAIL LROSSING ACCIDENT SEE DE 2203 OT JAN 76

$01508-C L 5$ $11709-000$ C1970-CLS $01571-005$ $01472-005$ $01 \times 73-005$ $41+74-005$ $1575-000$ $4+10-C u 4$ 01977-005 L 2 Cuz-0us 02046-006 ¿2uบ7-057 उ८Uि 8 -OCo 2u13-c0 $026<7-C 67$ $024<8-C C 0$ Cथट $30-500$ $02 \operatorname{cs} 1-C 05$ $02 \cos 3-004$ $\mathrm{C} 2 \mathrm{C} 36-0 \mathrm{O} 4$ $02 \cup 37-600$ C2038-005 $0=029-004$ $02029-004$ $2040-004$
$02041-304$ $02041-304$
$5<042-0044$ $02043-0 C_{4}$ $\mathrm{C}_{2} \mathrm{C} 44-0 \mathrm{O} 4$ $02 \cup 45-004$ 0ंC 78-014 CcC78-014 $02007-001$ $02 \cup 42-0 C 7$ C2CY4-CC5 บ2u96-001 [2Cy7-J01 C2112-013 -2115-014 $02116-010$ $0<116-010$ $0<119-001$ OCCU4-OCI

.... acceleration/time....... Intut.... transportatiun..

KEAK EAU CLLLISIUA AND DERAILMEMT MEEKEK, LA MAY $1 \times 75$

KAIL ACCICENT, HEAD-IN, EL MONTE, CA 2 AFR 1473

FAIL ACCIUENT, HEAD-ON, LUFKIN, TEXAS 18 NOY 1973

RAIL ALCIDEIT, HEAD-OA, COEDEA, ILLINOIS 2 DEC 1473 


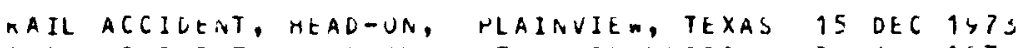
FAIL ACLIOENT, HEAD-ON, ST LUUIS, MISSOUNI CC JUN 197 KAIL ACCIOENT, HEADOON, MENPHIS, TEV 24 JAN 1475 KAIL ACCILENT, SIDE-ON, ERRLS, TEXAS ZQ NUV 1S74 KAIL ACCIDENT, OPEN SWITCH, WINLHAR, UHIC $2 \angle$ NUUV $1 \$ 73$ KAIL ACCIDENT, FAKIUG, LANE JUNCTION, NJ 17 JUN 1 Y 74 RAIL ACCIDENT, REAR EMD, HUKRICAINE, ALASKA 5 JUL 1475 RAIL ACCIDENT, REAR END, LEETUNIA, UHIO O JUN 1 , TS RAIL ACCIDENT, FEAR END, LUFO, ILLINOIS 3 JAN 1975

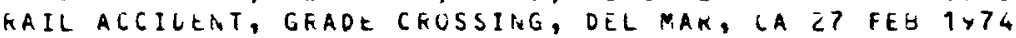
RAIL ACCIDENT, OKOKEN KAIL, THUMPSONTOWN,PA DEC 1474 RAIL ACCIUENT, GKADE CKCSSING, TKACY,CA G MAR 1575 RAIL ACCIDENT, GKADE CKOSSING, ELMOUD, IL 19 NOV $1 \times 75$ RAIL ACCIDENT, UAPRUTECTED ORADE LROSSIMG 7 FEO 1976

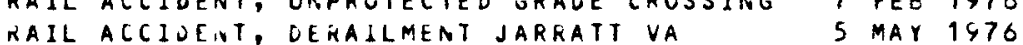
KAIL ACCIDENT, HEADOON G TRAIAS PETTISVILLE OH

AUTOMATLC WHELLUN-THE-GRUUND DEKAILMENT DETECTION SYS AUTOMATEL WHEEL-UN-THE-GRUUND DEKAILMENT DETECTION SYS RAIL CEOSSING ACCIDENT WITH TANK TRUCK SEE EDEZCZO CARITRAIN-FRUNI TO SIDE IMPACT UAPRUTECTED CROSSING SCHCOL BUS-TKAIN COLLISIUN STRATTON, NE 8 AUO 1970

TKAIN DERAILAEMT CAKKYING 2 CUNTAINERS UF RAM

KAIL ACLIDENT, GKADE CRUSSING, MAKLAND, UK 15 DEC 70 RAIL CRUSSIIÚ ACCIDENT WITH SCHUOL OUS LAFAYETTE, OH TRAIR, ACCIDENT, FAIL FAILURE, RELEASED ANHYOKOUS AMMON RAIL CROSSING ACCIDENT WITANKTRUCK THAYEK, MS 10 MAR 7 RAIL ACCILENT, REAR-END ÖLACK ROCA, NY RAIL ACCIUEAT, REAR-EAD NEW YURK CITY, AY Y Y JUL 75 FAIL OERAILMENT, FIKE, EXPLOSION FERTILE, MN 22 OCT 75 RAIL ACCIDENT, HEAD-OA CUAHOMA, MS Z NOS 75 RAIL ACCIUENT: HEAD-UN, SIATE-OF-ART TRANSIT 11 AUG 73 RAILACCIUENT, HEAD-UN, STATE-OF-ART TRANSIT IT AUG 73 RAIL ACCIDENT, DERAILNENT HASTINOS NE HAVEN IN OCT 70 RAIL ACCIDENT, HEAD-ON, 2 TKAIAS, VEW AAVEN IN $O$ OCT 70 KAIL ACCIUENT, OERAILMENT GOCDMAY FS AMTRAK

KAIL ACCICENT, RAIL FAILUKE, PENSHCOLA FL 8 NOV 77 RAIL ACCIDENT, KEAR-ENU BROUKLYN. INCTION WV C7 JAN 70 NAIL ACLICEMT, REAR -END SANEORN NI

RAIL ACCIDENT, JEKAILMENT WARD AR 27 JAN 76 3 FEB 70 KAIL ACCILENT, REAR-EMD TKACK CAK DINGLE IO 1 MAAR TO FAIL ACCIDENT, OERAILNENTIFIRE CALVERT TX ZC MAR 70 RAIL ACCIDEIVT, REAR-END GLAIRSVILLE PA 7 APR 70 RAIL ALCIDENT, SHIFTED LOAD HUGHES UH 7 JUN 76 RAIL ACCIUENT, DEKAILMENT CLIFFORD FI 10 JCT 76 RAIL ACCIDENT, AMTRAK UERAILMENT CHICAGU IL 18 OCT 70 FAIL ACCIDENT, GRADE CFOSSING GOLDEN MS I JAN 70
$014<8-003$ $619 \div 9-604$ $014=0-003$ C19:1-003 $01 \times 32-002$ $\mathrm{C1}+ \pm 3-\mathrm{CO} 4$ $01545-012$ $01946-014$ $01947-004$
$01948-003$ $01749-002$ $01950-015$ $01951-C 12$ $02 C 10-006$ $C<011-013$ C2012-048 02017-CCe $0<4<3-0 u z$ $0<C<9-041$ C2C $32-\cos$ 02034-006 $0<049-042$ $020>5-008$ 020 y $8-004$ 0र 1C9-012 C2118-004 $021<1-004$ Cद1ट2-004 $021<3-005$ Cट1<4-003 C2141-008 $0<141-008$
$C 2142-010$ C2 $143-010$ C2147-014 0ट909-023 $0<194-504$ $02195-005$ $0<156-006$
$0<147-005$ C2198-005 $021 \times 9-004$ ن22 $240-00 t$ $0<<01-003$ $022 \cup 3-006$ 
NATUKE GF SHIF CULLISILNS WITHIN FCATS

TANSHIP ACCIDENT, HENETKATIOMJGAS IIKE IN CAKGC TANKS

$C \leq \cup 6-C \cup 1$

10 JAN 74

$0204 c-001$

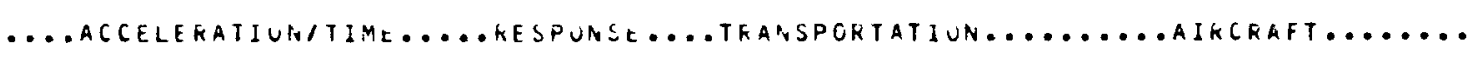

(IMPACT)

$(I N F A C T)$
PLLL SCALE CRASH TEST UF A CH-47C HLLICUPTEK

LIGHT HLANE CRASH IESTS aT IMPACT VEL.TS AND 27 FSEG

$C<\bar{C}<4-040$
$C 2100-354$

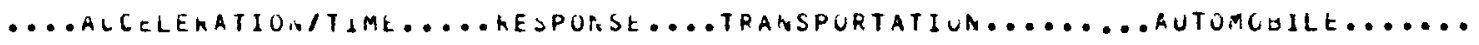

TKUCK ACCIDENTS, NHTSA, FINAL REPCRT

TRLCK ACCIO

TRUSK ACCIDENTS, NHTSA, VULC,NO.S

TKUCK ACCILENTS, NHTSA, VOLO,NO.O

TRUCK ACCIDENTS, NHTSA, VOLC, NOO.7

TKUCK ACCIDENTS, NHTSA, VULC, NO.O

TEUCK ACC IUEMTS PHTSA, VULO, HO. Y

CAR ACCICEAT, MHTSA,VOL.O, HO 11

TKUCK ACCIUENT, NHTSA, VOL.6, NO.1

TRUCK ACCIDEAT, NATSA, VOL.6, NO.1C

TRUCA/PICKUP TRUCX ICAMPLR AND TKAILER CULLISIUN

AUTO COLLISICN WIANL COLLAPSE OF ORIDGE, SILOAM, WC

TKUCK ACCIDENT, EXPLOSICA IFIRE, EAGLE PASS, TEXAS

SCHOOLOUS / AUTO CCLLISICN/FOLLOVER, ASHLANU, OREGON

CULLISION OF TRUCK AND OUS, NEAR HAMILTON, GA JUN 1 Y75

CULLISION OF WINNEEAGO MOTOR HOME WIBRIDGE 1 JUL 1475

LOLLISION OF TKUCK AND CAK, LOVINGTCN, NM 3 JAN 1970

CAR COLLISION. FRONT-TO-FRONT IMPACT TEST AT GU MFH

TRUCK TRACTOR-TRAILER/GKOUNU/KOLLUVER VUL 7, NO 1

TRUCK TRACTUR-SPORTS VAN IMFACT VOL 7 , WO 1

TKUCK CAF ALCILENT, BALTINOKE, MD, ON INTERSTATE

TKLCK CAF ACCIOENT, KEAK END UNCEK-FIDE IMPACT, VOL 7

GASCLINE TKUCK ACCIUENT, SEATTLE, WK

4 DEC 1975

IFACTOR TFAILEK/CAR/SCHCOL IUS, KEAK END, ROLLOVEF

CAK OUS ACCIDENT, OVERLANL MARK, KAMSAS 7 OCT 1975

STATION WAGONITRAIN ACCIDENT, GALTIMORE, MD DEC 1 Y75

TKACTOK TRAILEK, REAR ENU AND KULLOVER AY 1 JUL 1975

TFUCR ACCIDENT, TIFE ELEW OUT, IMPACT, NY 21 JUN 1975

STATIGN HAGON/SCHCOL BUS, HEAD ON, HAMBLRG, NY SEP 1975

TRUCK/SCAOOL OLS/TRAIN ACLIUENT, ARKANSAS 11 SEP 1475

TRACTUK TFAILEK, FRONT TU SIDE IMPACT F 1975

TRUCK CAF ACCIUEAT INTEKSECIOM, OALTIMOKE,MD FEJ 197 D

CAFIPULEITKAIN SIDESWIPE IMFACT,LUS ANGLES C 5 SEP 1 Y 75

TRLCK CAR ACCIDEAT,KEAK END, SAR DIE UO FKEEWAY JAN 1970

TRUCK ACCICLAT, TKI-STATE RIG, GILA EEAU AZ

TRLCK ACCIUENT, CULLISICA LEFT ROADWAY WASHIAGTON OC

IRUCK/SCHOOL OLS COLLISION LAKE PLACID FL

ThUCKIVAR/AUTO ACCIUENT LEXINGTON VA
C1075-014

$01070-003$
C1ะ77-0u4

C1E77-0U4
C1\&78-008

C1879-6uo

01ठ80-011

$01801-014$

$01898-005$

C18 $19-005$

$01 \times 00-004$

$01 \times 01-015$

$01952-014$

01853-011

61754-017

$01555-005$

$01956-015$

$01957-060$

$01 \times 58-002$

$01902-004$
$01903-005$

$01404-006$

$01905-906$

$01706-001$

C1967-205

$01508-005$

C1909-CC6

$01570-005$

C1971-6C5

$01472-005$

$01573-\cos$

$01 \times 74-000$

C1975-LEE

$01976-0104$

$21977-605$

C<OU6-0CO

CCUU7-CU?

C2こし $8-0 C t$ 
LUA UER TRUCK ACCILENT-CARLO SHIFT, MARTFORD O OEC 1975 TRUCK/TKAIV CKUSSIAO ACCICENT AYY ROLTE 1 Y 10 VOV 1 Y 73 TRUCK, FKCNT COKAEK IMPACT, CCNCUKD, ANY 27 VUV 1ST3 TRUCK HEEL/CAK - TLP IMPACT NY DTATE 20 NOV $1 \times 73$ CARITRACTUK-TKAILER UNDEKKIDE CCLLISION 12 DEC 1574 IRACTUK-TKAILEK LAMFIAU ACCIDENT, HARDIIV, KY JUN 1475 MOTOF MUME ORAY-CFF KGADMAY, IMHACI OFIRE OEXPLOSIUN 9575 TKACTOR TTKAILEKACAR S TKACTOR THKAILEKICAR SICESWIPE KURAL KY 17 AUG 197 CIDENT PACIFIC COAST HIGHWAY 17 JU' 147 WhITE FKE ISHTLINER ACCIUENT VENTURG FRELWAY JUN 1975 TRUCK TKAILER NOLLOVEK, FUOK EKAKES, VEK.Y., CA JUIN 1975 TRUCK CAR ACCILENT, LANE CHANUE, LOS ANGELES JUN 1575 TAAKTKUCK ACCIUEI,T, HELIUM FUEL LEAKAGE JUL 1Y75 JACKIVIFING TKAILEK FOKCED TRAGTUR INTO ORIDUE JUL 1Y7S TEUCK TMAILEK ACCIDENT, CARGO SHIFT, KOLLOVER AUU 1975 TRUCK ACCIDEVT, PFOPAIY, OVERTUKN ANO FIRE Y MAK $1 \$ 72$ CAR/TKAIN, ORADE CFUSSING, DES MOINES,IOWA SEE DO ZCES TRACTOK TRAILEK COLLIDEV IITH CAK GAXTER, CA 11 MAR 77 TRACTOR/CAFOO TANK TRAILEK ACCICENT, CHERRY HILL, NJ TANKTRUCK/RAIL GRADE CROSSING ACCIDENT SEE DB COY5 SCHUOL OUS TRAIA GRADE LRLSSING ACCIDENT SEE DE 2098 TRACTOK-SEMITRAILER COLLISICN MULTIPLE VEHICLES AUG 76 MINI MUTOR HOME/CAR ACCIDENT MCALESTER,OK 14 JUL 77 TRUCK ACCIDENT, PENETKATED ERIDGE RAIL RELEASEO HM CG TAMRTRUCK RAIL CKOSSIAG ACCIDENT SEE DB2118 10 MAR 75 TRUCK ACCIDENT, GASULIAE FIFE, EEATTYVILLE KY OS/24/77 TRUCK RAIL CROSSING ACCIDENT SEE DE ZEUS 27 JAN 70

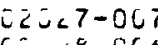
$C \hat{<}-\angle 8-C Q 6$ $0 \leq 530-006$ Ċus1-cos $0<233-0 u 4$ $i<0 \pm 0-004$ $0=037-006$ CदCs8-005 $02038-005$ $02539-004$ $\mathrm{C2C} 4 \mathrm{C}-\mathrm{CO}$ C<ट41-0u4 $02042-604$ $02043-604$ $62344-004$ $02045-064$ $0<278-014$ $0<007-001$
$0<0<5-007$ $0<007-001$ $C<\cup>2-007$ $02096-001$ C $\angle C=7-0 C$ 02112-013 02115-014 02116-010 02119-001 $02108-014$ $C \bar{C}<\cup 4-O C 1$

(IMPACT)

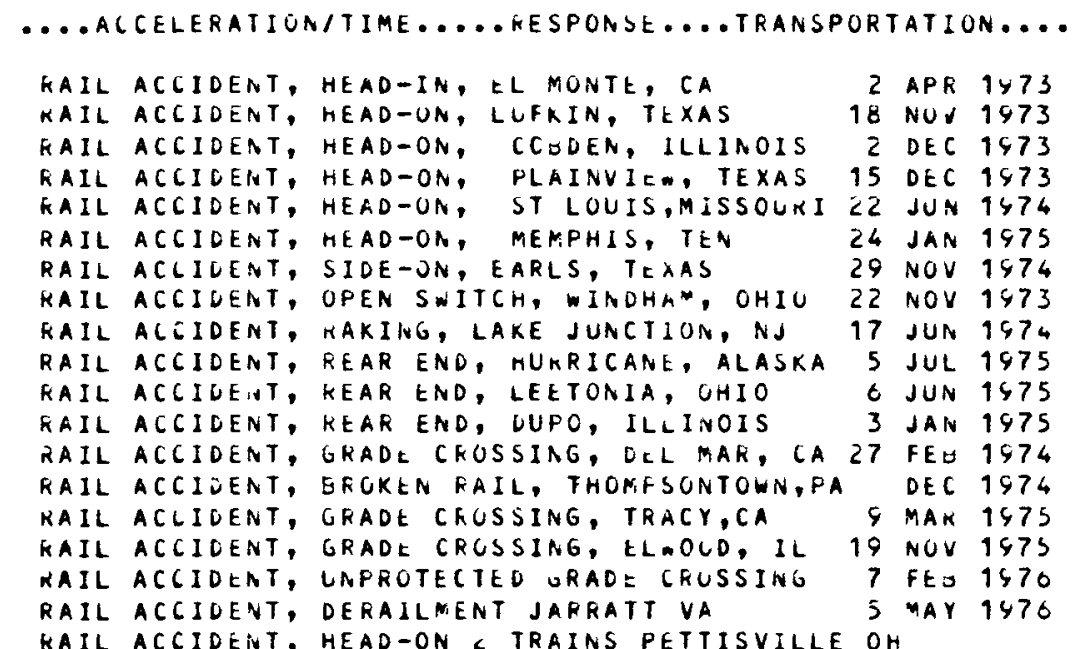

$01925-064$ $019<6-003$ $019<7-003$ $019<8-003$ $013<8-003$
$014<9-004$ $014<9-004$
$01830-003$ $01930-003$
$01931-003$ $01 \times 32-002$ $01433-304$ 01945-012 $01840-514$ $01940-014$ $01947-004$ $01948-003$
$01449-002$ $01550-015$ C1851-012 C<U10-000 $02011-013$ $02012-002$ 
(IMFACT)

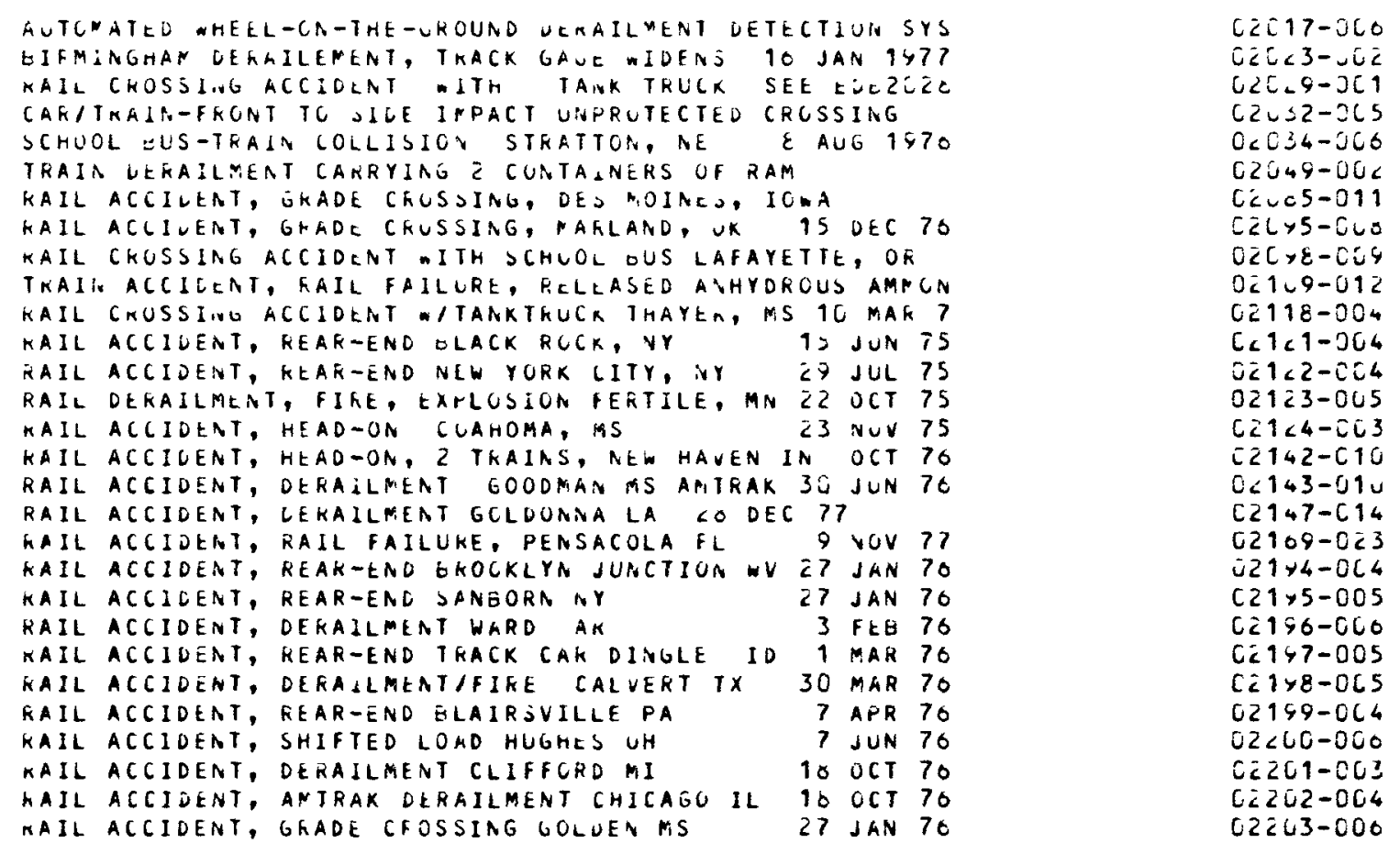

.... accelekationttime..... kesponse.... transportation........... Ship........

NATUKE OF SHIP COLLISIONS WITHIN POETS

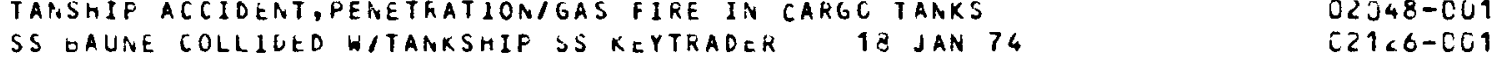

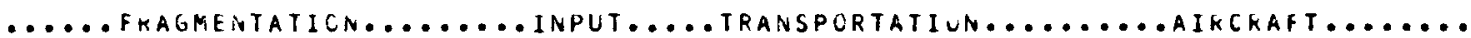

HELICOFtek LANGING lear CULlaPSED PAN AM ELOG NY 0ZO46-001 HELICOFTEK MAIN KGTCK HUD TKUNNIUIN FRACTUREDISEPAKATED O O2CAT-CO1

..... Fragmentation.......

HELILOPTER LANUIQG GEAR CULLAPSED PAN AM ELDG NY CZUGO-OU

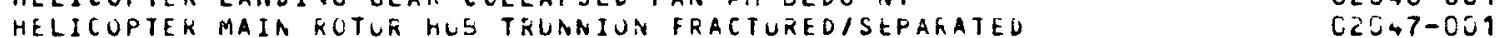

AIRCEART ACGIDENT, INFLIGHT SEPAKATION PROPELLER GLADE CLCUG-CLI AN ASSESSMEIT OF TECHNCLOGY TUKECJET ENUIAE FAILUEES C<139-3U1 
(PUNCTUKL)

(PUNGTLKE)

(PUNCTURE)

( $P U N(T U R E)$

(PUN(TUKE)

(PUNCTURE)

(PUNCTURE)

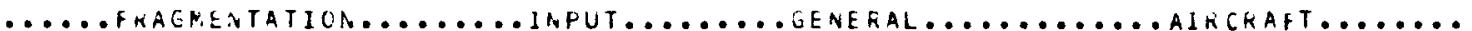

TUFEIN ENLIAE KUTOR OISK FAILURES 1962 TU EAKLY 1973

C2us4-013

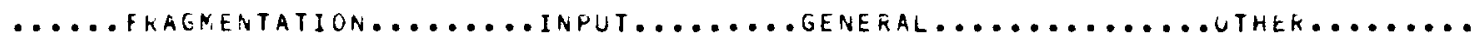

FRAGIENTS, MASS, VELOCITY, STARDAKD ARTILLEKY SHELLS

$61614-002$

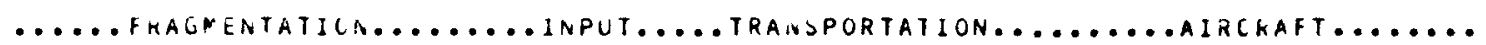

ROTOK FKAGMENT CUNTAINMENT-JET ENGINE FAILURE - 1×7C ROTOR FAILUKES, GAS TUREINE, US COMM AVIATION 1973 KOTOK FAILUFES, GAS TUREINE, JS CUMM AVIATION 1474

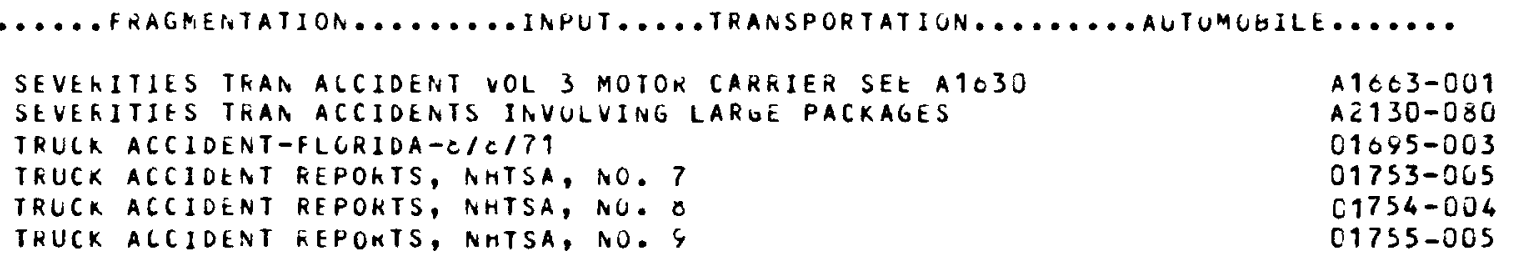

$01755-005$

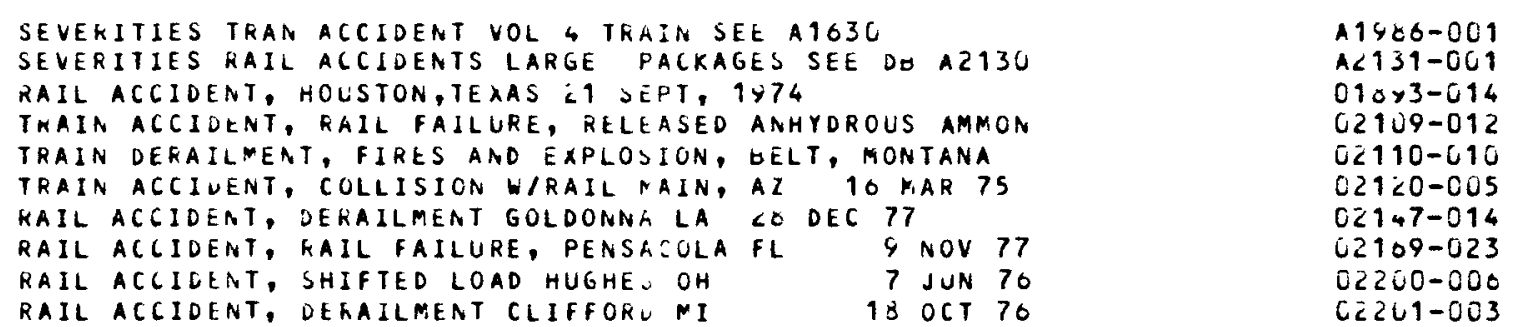

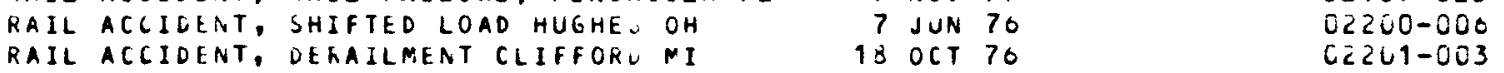

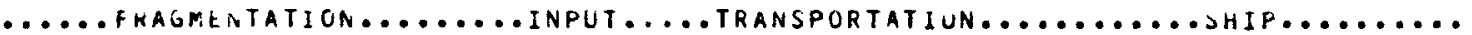

IMPACT, FENETKATICN, SHIP COLLISICN AND GROUNDING 01550-001 SS EAUNE CULLIDED WITANKSHIP SS KEYTRADER IE JAN $74 \quad 021 \angle 6-001$

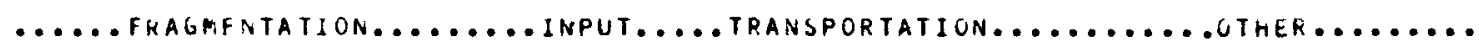

SEVERITIES UF IRANSPORTATLON ACGLUENTS VUL 1 SUMMARY A1020-000 
(FUNCTURE)

(PUNCTURE)

(EXFLOSION)

$(\operatorname{EPLOSION)}$

$(E X P L U S I O A)$

(EXFLUSION:

(EXPLUSIOA)

(EXPLUSION)

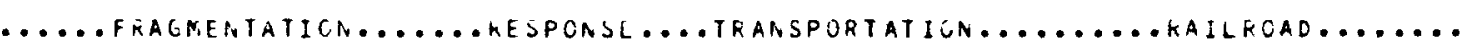

CARSU KESPU.NDE TRUCA ANE KAIL ACCCIDENTS SEE EOG1080

HAIL ACCIOENT, HCUSTGN, IEAAS, 19CCT1B?1

RAIL ACCILEAT, CRESCENT CIIY, 19AUUIY?O

KAIL ACCICENT, HOUSTON,TEXAS 21 SLPT, $1 \times 74$

THAIA ALCIUEAT, HAIL FAILURE RELEASEU APHYDROUS AMMON

TKAIN DERAILNEINT, FIRES AND EAPLOSIUN, OELT, MONTANA

TKAIN DERAILNENT, FIRES AND EAPLOSIUN, OELT, MONTANA

TRAIA ACCIDENT, CULLISIGN W/RAIL MAIN, AZ 10 MAR
RAIL ACCIOENT, DERAILMENT GULDONMALA LE DEC T7

KAIL ACCIDEIT, KAIL FAILUKE, PENSACCLA FL 9 NUV 77

KAIL ACCILENT, SHIFTED LOAD HUGHES UH 7 JUN 70

FAIL ACCIDENT, DERAILMENT CLIFFOR̃ MI

$010 \times 9-002$

C1C46-Cue

$01047-009$

$010 \times 3-014$

$021 \times 9-012$

$02109-012$

$02110-010$

$021,0-005$

$02147-014$

$0<109-0<3$

$02200-000$

IO UCT 70

......fkagmentation.......

SS GAUNE CULLIDED WITANRSHIP SS KEYTRADER IE JAN 74

$021 \div 6-001$

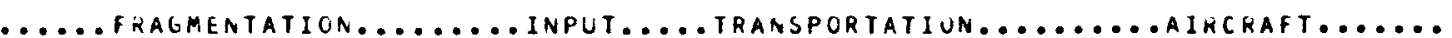

AIFCKAFT ACCIDENT, EOEING 707-331甘 OE SEP $74 \quad 02154-044$

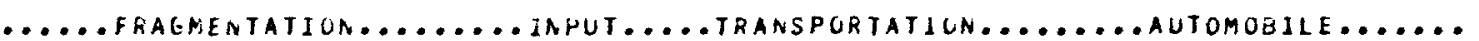

TRUCK ACCIDENT-NEW JERSEY $9121 / 72$

TRUCK ACCICENT-GEORGIA-O/4/71

TRUCK ACCIUENT-VIKGINIA-3/9172

KISK ASSESSMENT GF TRANSFURT UF LFG

$010 \times 0-003$

$01644-003$

$01046-003$

Cं́177-091

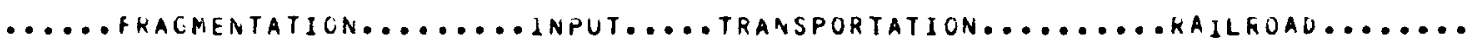

KAIL DERAILMENT, FIKE, EXFLCSION FERTILE, MN ¿Z OCT 75

$0<1<3-305$

.......

EXFLUSIUN FIRE EAUULFEL THE MIT ELIAS, UNLOADINO APR $74 \quad 021<7-C L \overline{3}$ KISK ASDESSMENT OF TRANSPORT OF LNG SEE EDE $\angle 177 \quad 02178-001$

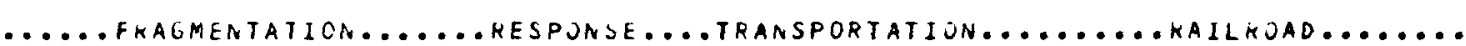

FAIL DERAILMENT, FIKE, EXFLOSICA FEKTILE, MN Z2 OCT $75 \quad 629<3-005$

...... fkachientation.......

EXFLUSIOA Fite ENGLLFED THE M/T ELIAS, UNLOADING AFF 74

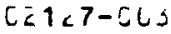




\begin{tabular}{|c|c|c|c|c|}
\hline $\begin{array}{l}\text { TYFHOON } \\
\text { TYPHOUN }\end{array}$ & $\begin{array}{l}\text { FEFUKT } \\
\text { PEPOFT }\end{array}$ & $\begin{array}{l}\text { - FACIFIC } \\
\text { - PALIFIC }\end{array}$ & $\begin{array}{l}\text { UCEAN }-1950 \\
\text { UCEAN }-1900\end{array}$ & $\begin{array}{l}C 1002-0) 3 \\
010 \cup 3-0 \cup 5\end{array}$ \\
\hline TYFHCUN & REPUNT & - FALIFIL & OCEAN-1961 & $510_{4} 4-303$ \\
\hline TYPHOON & KEFUKT & - FACIFIC & OCEAN - $19 C Z$ & C1Eus-CO4 \\
\hline TYPHOUN & KEPOKT & - PACIFIC & GCEAN - 1963 & $01006-003$ \\
\hline TYPHOON & FEFOFT & $-F A C I F I C$ & UCEAN - 19CS & $C 10 \cup 7-C C^{3}$ \\
\hline TYFHUON & KEPOKT & - FACIFIC & UCEAN -1906 & $01008-0 C j$ \\
\hline TYPACON & REFONT & - FACIFIC & OCEAN - 1967 & 010ட9-CL: \\
\hline TYFHUON & KEPOKT & - PACIFIC & UCEAN-19EE & $01210-00$ \\
\hline TYPHUON & REFORT & - FALIFIC & UCEAN - 19E9 & $01 \approx 11-002$ \\
\hline TYPHCON & KEPOHT & - PACIFIC & -1970 & $c 1=12-003$ \\
\hline TYPHUON & FEPUNT & - PACIFIC & CCEAR - 1974 & $c 1=13-3 c_{3}$ \\
\hline TKOF ICAL & L CYCLON & its & & $01 \div 56-3.2$ \\
\hline
\end{tabular}

(CKUSH)

(CKUSH)

( $C R \cup S H)$

( $C$ KUSH)

( $C R \cup S H)$

(IMMERSION)

(IMMERSION) 
(IMPEKSION)

$(I M P C K S 10 \mathrm{~N})$

(IMMEKSION)

$(E X P L O S I O N)$

$($ EXPLSIUN)

(EXPLOSION)

(EXFLUSION
-........PRESSURE.

SIAKINU SS SILVEK DUVE, GRACAED WALL IN GARGO HCL

JIAKIVG GREAT LAKES GULK LAKUU VESSEL SJ ED FITZUEKALD

Eடบ49-0し3

$621<5-001$

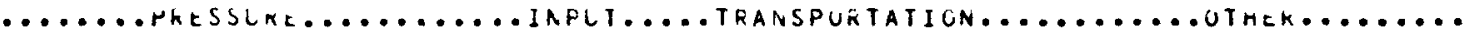

SEVEKITIES UF TKANSHOFTATION ACCILENTS VUL 1 SUMMARY

$A+30-060$

........ Pressufe.........

SIRKINO SS SILVER DUVE, CMACKED WALL IN CARGO HOLD

$02609-003$

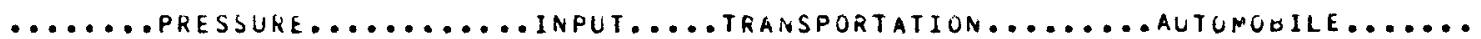

HYDKOGEIY TRANSFORT AND HATHDLING EXPLOSIÉ.

TFUCK ALCIOENT, EXPLUSIUNIFIRE, EAGLE PASS, TEXAS

$017>8-601$

$01953-011$

CCLLISIUN OR WINIEGAUO MOTOK HOME WIERIUUE TOJUL 1975

$09 \div 56-015$

YOTOK HUME, RAN-OFF KOAD.AT, IMPACT,FIRE, EXPLOSIUN 1§75

$-2037-000$

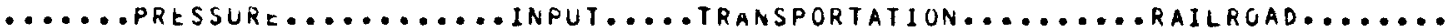

KAIL ACCILENT, EAST ST LOUIS, ILLINUIS - $1 / \angle 2 / 72$

RAIL ACCIDENT, CRETE, AEEMASKA - C/13/64

$01706-C C_{3}$

RAIL ACLIDENT, HOUSTON, TEXAS - 1U/1Y/71

$01747-003$

FAIL ACCIOENT, JECATUR, ILL 14 JULY,1474

KAIL ACCIUENT, EENSON,AK Z4 MAY,IG73

RAIL ACCIOENT, HOUSTON,TEXAS $\angle 1$ SCPT, 1Y74

RAIL ACCIDENT, EXFLUSIUN OF PRM, WENATCHEE,WI AUG $1474 \quad 01 \times 38-035$

C1589-31

$01891-C 10$

$01843-014$

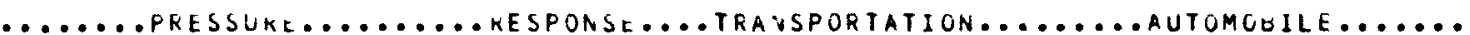

$\begin{array}{lll}\text { TEUGK ACCIDENT, EXPLOSIONIFIRE, LAGLE PASS, TEXAS } & \text { O1Y53-011 } \\ \text { COLLISION GF WINNEBAOO MOIOR MUME WIBRIDOE 1 JUL 197S } & \text { C19S6-U15 }\end{array}$

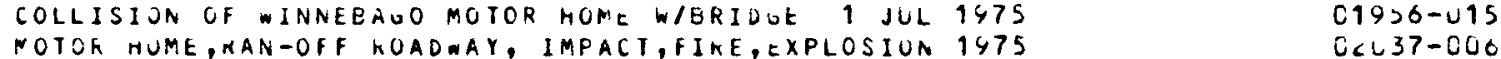

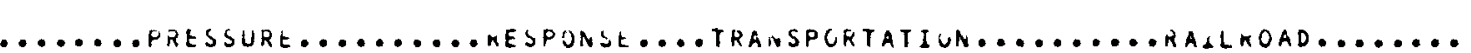

WAIL ACCIDERT, OECATUR, ILL 14 JULY, TY74
HAIL ACCIUENT, OENSON,AR 24 MAY, IY73

KAIL ACCIDENT, HOUSTON, TEXAS C 1 SEPT, 1974

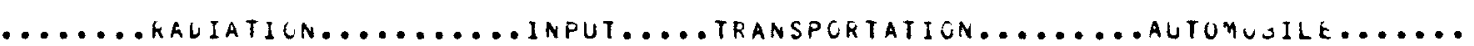

TKAN ACCIDENT KISKS IN RULLEAK FOAEK INO 1975-2LCU 
........

IRAN ACGIDENT MISKS IN NLCLEAK FOnER INL SEE EDO 19EG

$01991-001$

(LIGHTNING)

(LIGHTNING)

$(C K \cup S H)$

$(C K \cup S H)$

$($ C R SH)

$(\mathrm{CH \cup SH})$

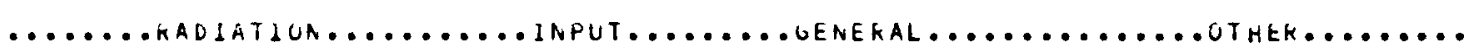

VOLTAGE ANC CUAKENT CHAKACTERISTIGS OF LIOHTNIVG STRIKE OC404-002 PKOPERTIES OF LIGHTNING SIRGRES $\quad$ 00999-C01

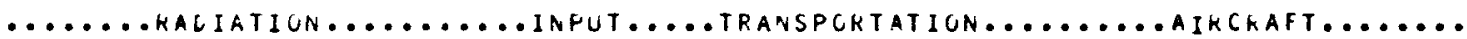

LIGHINII.G THREAT IO AIKCRAFT O1000-00E LIGHTNING HAZAKD TO AIRLKAFT MEASLREMEAT LIGHTINU INUUCED VOLTAGES IN AN F4H-1 CE 185-130

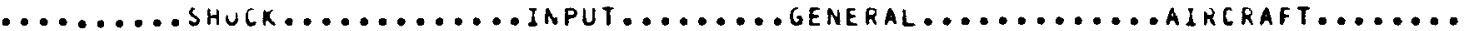

CFLSH ENVIRONMENT SMALL CUNTAINERS CARRILD OY US JETS $02114-012$

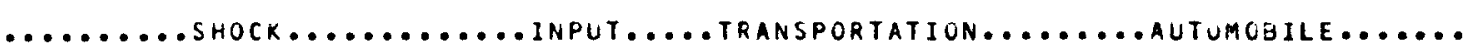

SEVERITIES TRAN ACCIDENT VOL 3 MOTOR CARRIER SEE A1630 A1603-001 SEVERITIES TKAN ACCADENTS INVOLVIMG LARGE PACKAGES A

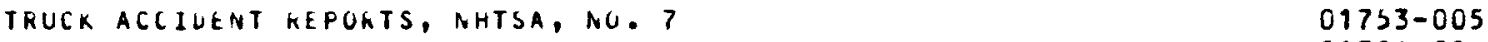

TRUCK ACCIDENT REPORTS, NHTSA, NO. O $\quad 01754-004$

TFUCK ACC JOENT REPORTS NTTSA, NO 5

TRUCK ACCIOENT REPORTS, NHTSA, NO:

TRUCK ACCIDENT, NHTSA, VUL.6, NO.1U $\quad$ 01899-005

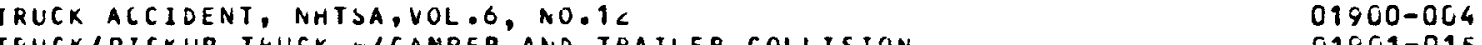

$\begin{array}{lll}\text { IRUCK/PICKUP TKUCK W/CAMPER AND TRAILER COLLISION } & 01901-015 \\ \text { IRUCK TRACTOR-TRAILER/GKOUND/ROLLOVER VOL 7. NO } 1 & 01962-006\end{array}$

$\begin{array}{llll}\text { IRUCK TRACTOR-TRAILER/GKOUND/ROLLOVER VOL 7, NO } 1 & 01962-004 \\ \text { IRUCK TRACTOR-SPORTS VAR IMPACT VOL 7, NO I } & 01903-005\end{array}$

TRUCK CAR ACCILENT, GALTIFOKE, MD, ON INTERSTATE

TKUCK CAE ACCIDENT, REAR ENU UNUEK-RIDE IMPACT, VOL 7 $\quad$ C1965-006

LUMEER TRUCK ACCIDENT-CARGO SHIFT, HARTFOFD O DEC 1975 $02027-007$

TRUCK WHEEL/CAF - TCP IMPACT NY STATE 26 NOV 1973 $02039-005$

TRACTOR-TKAILER CAMHING ACCIDENT, HARDIN, KY JUN 9975 C2US6-CO4

.........SHOCK............. INPUT..... TRANSPORTATION.........RAILKOAD.......

SEVERITIES TRAN ACCIDEAT VOL 4 TRAIN SEE A103i A1966-CO

SEVERITIES RAIL ACCIDENTS LARGE PACKAGES SEE DO A2130 A2131-001

RAIL ALCIDENT, HOUSTON, TEXAS L1 SEPT, 1974 C1893-014

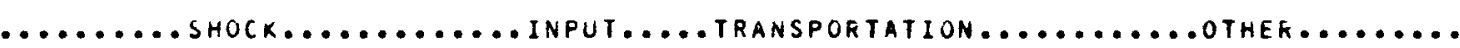

SEVERITIES OF TRANSPORTATION ACCIUENTS VOL 1 SUMMARY

A1030-30t 
TRLCK ACCIOEAT, AHTSA, VULE. N, NO.1U

C10 $10-045$ TKUCK ALCIDEAT, AHTSA,VOL.E, NC.1.

IFLCK/PICKUP TKUCK HILA:PEK ANE TKAILER COLLISIOV

TRUCK TKACTUK-TKAILEK/LROLND/KOLLCVER VOL 7 , NO 1

THUCK TRACTJR-DFORTS VAI. IMFALT VUL 7 , INU 1

TRULK CAK ACCIUENT, GALTIPORE, DD, ON INTERSTATE

$01901-015$

$019 \cup 1-315$
$01902-0 C 4$

TRUCK CAR ACCIDEAT, GALTIFORE, R. ON INTERSTATE

$01 \times 03-505$

T. VOL 7

LUNGEF TKUCK ALCILENT-CAKGO SHIFT, HAKTFURD O DEC 1Y7S

IRUCK WHEELICAK - TCP IMIPACT AY DTATE 60 NOV 1973

$01404-600$

$1705-600$

C1705-606

THACTOF-TRAILEK CAMHING ACCIDEAT, HARLIN, KY JUN 1975

C20 $21-005$

LC $30-034$

( $(K \cup S H)$

(IMPACT

(IPHACT)

(IN,FACT)

(IFPACT
RAIL ACCIOENT, HOUSTON, TEXAS, TYUCT1S71

RAIL ACCIOENT, HOLSTON, TEAAS CI SEPT, 1Y74
C1840-0Cs $11247-609$

$010 \times 3-014$

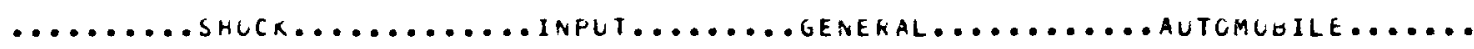

TRUCK ACCIDERT SFEELS A - SUMMARY

STATISTICAL STUOY OF TEUCK ACCILENT SPEEOS

$01607-002$

$01048-012$

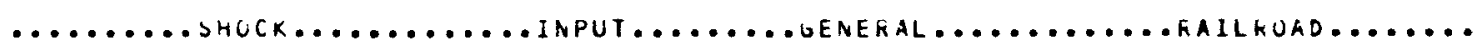

FREIGHT TKAIN ACCIDENTS, NUMEEK OF CARS INVOLVEO

$017<9-007$

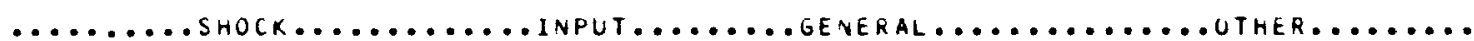

APFFOXIMATE DUFATION OF SOME SHGKT ACCELEKATION LOAOS

$00754-501$

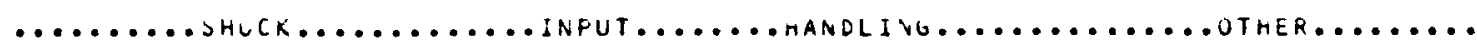

IMPACTS DUE TO LAKGE UROPS WHEN LUALING

MAXIMUM DROPS, PACKAGES IN TRANSIT

$[0242-001$ C16 16804

......... SHOCK............ INPUT.....TRANSPURTATION.........AIRCRAFT.......

SEVEKITIES TKAN ALCIDEAT VOL L CARGU AIKCRAFT SEE 1 C3U

AAALYSIS UF OINECTIUN OF LOADS I AIRCRAFT ACCIUENTS

ACCELERATIUN OH FLOCR-AIRLRAFT CKASHES

ACCELERATIOA, AIRCKAFT CKASH-VAKIUUS AAGLES OF IMPACT

LMAST TEST, LCOT AIRLIKAFT

CKAST TEST,CONDTELLATIUN AIKCKAFT

CIVIL AVIATION ACCIDENT DATA - 1 DE2 TO $1+72$

ANALYSIS OF CARGC A/C CKASH RECORUS CAE DATA

A $1002-C u 1$ $057>1-001$ $00752-001$ $40753-005$ C $11 \cup 4-0 \cup 7$

C11 $115-510$

$01004-C E y$ 
CFASH IMFACT CGIODITIONS, MULTI ENUINE TKANSPORT A/C SURVEY UF GIC LKASH SURVIVAEILITY, CAKGU AT A/C CG ACCIDEVT CATA, MULTIENGINE AIKCKAFT A/C ACCIDENT ULE EZ SEFT 1970

A/C ACCICEDI MARTIN 4 C OCT 197U

A/C ACEICENT CUNLAIN SCL DEC 1GC

A/C ACCIOENT CEMAVILLAND UHC IE FES $197 \mathrm{~J}$

AIC ACCIDENT DOLGLAD CSLO AFRIL 1 Y $7 C$

A/C ACCIDENT OC $\triangle$ JAN ISTC

$A / C$ ACCIOEMT DCG AYY 1676

A/C ACCILENT SKYVAN SERIES E JULY 1YTJ

AIC ACCICEAT DCQ 3Z SEPI 1770 SEE EDE 2159

A/C ACCIDENT DCE ESF SEPT 1970

AIRCKAFT ACLIDEIUT - DOEING 707 - 1/16/74

AIRCRAFT CRASH ENVIKONMENTS - NAVY

AIRCKAFT ACCIUENT - CUNVAIR 600-9127173

AIFCRAFT MIDAIK COLLISIUAS

AIRCFAFT DITCHING INVESTIGATION

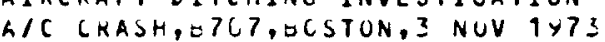

a RISK AND COMPARATIVE anaLYSIS OF NAVY AIRCRAFT DATA

AFMY ROTAKY WING AIRCRAFT ACCIDENTS-COST STUDY 1970-71

FULL SCALE CRASH TEST OF A CH-47C HELICOPTER
$(107 C-\bar{U} \overline{C Y}$

C1671-

$01072-C 1$
$01076-C 43$

$01077-003$

$09678-603$

$01019-003$

$01000-503$

$01000-50$
01000

C1602-0us

$01003-003$

$01604-003$

$01006-003$

$01800-001$

$01019-001$

$010<0-001$

$01825-005$

$01822-002$

$010<3-002$
$01843-0<3$

$01843-0<3$
$01857-001$

$010 \times 5-010$

$020<4-040$

ACCELEKATIONS, AUTOMUOILE (RASH (HEAD-ON)

ACCIDENT REPORT, TRLCK - VENTURA, CALIFORNIA, 8-18-71

TEUCK ACCIDENT-NEW JERSEY $9 / 21 / 72$

TEUCK ALCIDENT-ARIZUNA O/ $11 / 70$

TKLCK ACCIDENT-VIRGINIA $4 / 93 / 72$

TRUCK ACCIUENT-PENN-Y/5/71

TRUCK ACCIDENT-GEORGIA-0/4/71

TRUCK ACCIDENT-FLORIDA-C/O/71

TRUCK ACCIDENT-VIRGINIA-3/9/72

TRUCK ACCIDENT-TENN-5/13/72

RAIL/KOAD VEHICLE ACCIDENT, OKLAHGMA - 4/5/79-SEE 1710

RAIL/ROAD VEHILLE ACCIOENT, NE YUEK $-3 / 24 / 7 \overline{2}$

RAIL/ROAD VEHICLE ACCIDENT, ILLIAUIS -1/24/70-SEE 1714

TRUCK ACCIDENT REPOKTS, NHTSA, NO.?

TRUCK ACCIDENT KEPORTS, NATSA, NU.

TRLCK ALCIDENT KEPORTS, NATSA, NO: Y

TRUCK ACCIDENT REPOKTS - GOSTON UNIV., 1473

TKUCK ACCIDENT REPORTS - NHTSA, NU. 3

FAIL-HIGHWAY GKADE (ROSSING ACCIDLNTS, 1772 (SEE 1775)

RAIL-HIUTHAY GKADE (RUSSIAG ACCIDENTS, 1973 (SEE 1777)

TRUCK ACCIDENT - TENNESSEE - E/27/73

A $2130-080$

$00738-0 \mathrm{C}$

$01059-003$

$01691-003$

$016 \times 1-003$

$016 \times 2-003$

$01043-003$

$01644-003$

$01645-003$
$11046-003$

$01097-00$

01711-001

01712-003

c1715-001

$01753-005$

C1754-004

$01755-005$

$01770-064$

$01771-003$

01776-001

$01778-O C 1$

01703-0u1 
TKACTOK-SEMITKAILER COLLISICN MULTIHLE VEHICLES AUG 73 - IAI MUTOR HUME/CAR ACCIDENT MCALESTER, UK 14 JUL 77 TKUCK ACCIDENT, FENETKATEU ERILGE FAIL KELEASED HP, CG TAAKTRUCK RAIL CAUSSINE ACCIDLAT SEE OE T11E 10 MAK 75

TKUCK FAIL CROSSING ACCIDENT SEE DE $22 Z 3$ Z 7 JAN 70
Uட் $112-013$ C⿺ $115-014$ C¿ $116-010$ $0<119-001$ CZ2ட4-DU1

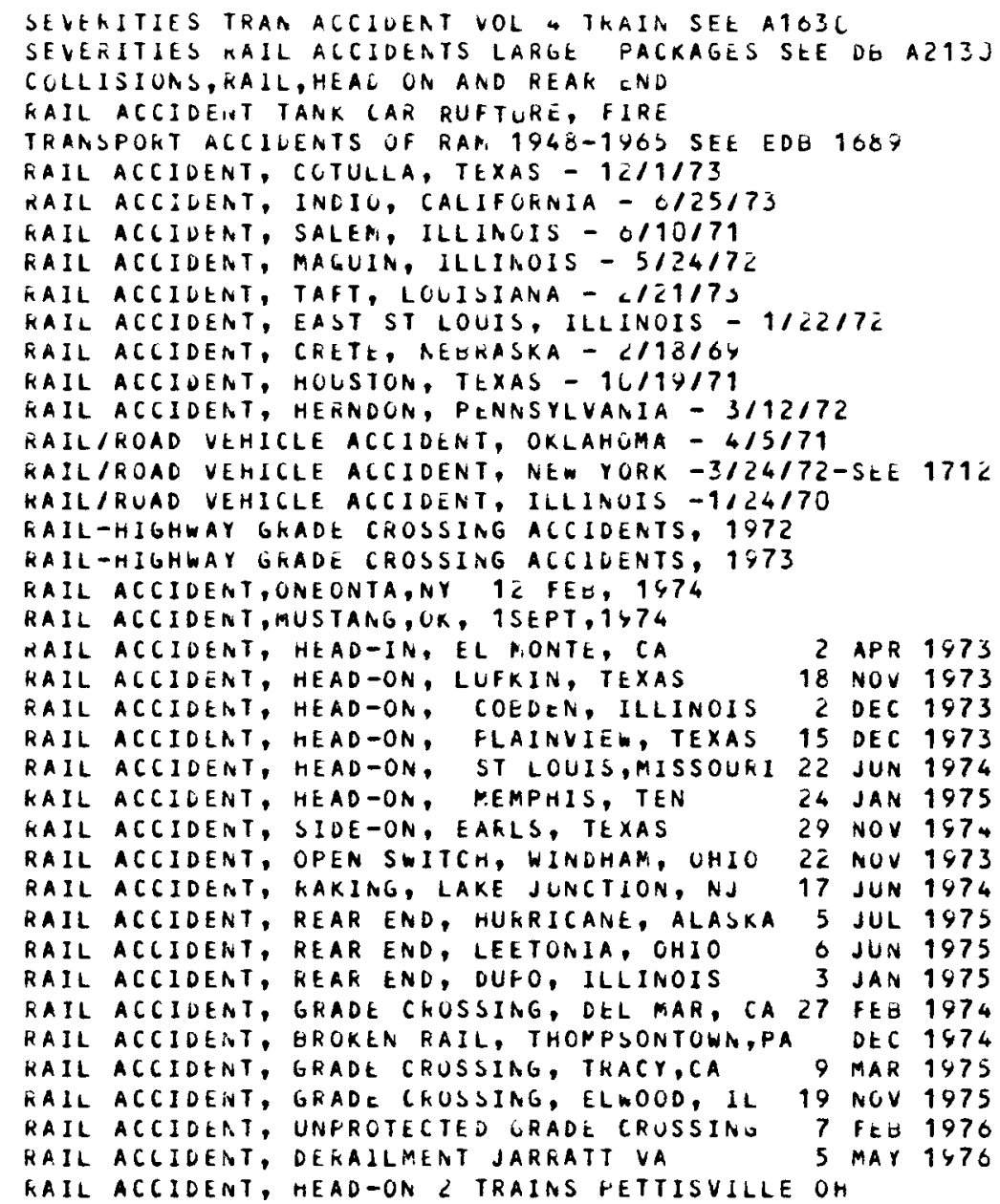

A $1800-001$

A $2131-001$ $01005-000$ $01075-$ Use $01700-011$ $01701-001$ $01701-001$ $017 \cup 2-001$ $01703-002$ $01704-001$ $01705-006$ $017 \cup 6-003$ $01707-003$ $01708-010$ $01709-003$ C1710-002 $01713-001$ $01714-002$ $01775-002$ $01777-002$ $01840-012$ C1842-010 C1842-010 $01925-004$ $019<6-003$ $01927-003$ $019<8-503$ $019<9-004$ $01730-003$ $01931-003$ $01932-002$ $01932-002$
$01933-004$ $01933-004$
$01945-012$ C1946-014 $01947-004$ $01948-003$ $01949-002$ $01949-002$ $01950-015$ $01951-01<$
CCS10-006 Cc011-013 परि12-000 02017-000 


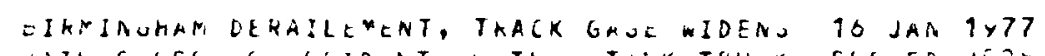
KAIL CKCSSIVG ALLIDENT ITA TAINK TKULK SEE EDLLCZO CAR/TRALA-FKONT TU SIDE IMPACT UNFROTECTEO CKOSSING SCHOCL OLSTTKALN COLLISION STRATTONA DE FAIL ALLICEAT, GRADL CKUSOIAG, DES MOINGS, IOHA

RAIL ACLICENT, GRADE CRUSSING, MKRLAND, UK 15 DEC 76 AAIL CRUSSIVG ACCIOENT ITH SCHOUL EUS LAFAYETTE, OK TRAIN ACCICENT, KAIL FAILUFE, RELEASED ANHYDRCUS AMAON TEALN UEKAILMENT, FIRES AND EAPLUSIUN, OELT, MUNTANA KAIL CKCSSINC ACCIDENT WITANKTKLCK THAYEK, MS TU MAK 7 TRAIA ACCIDLAT, COLLISICN WIKAIL MAIN, AL 1O MAR 75 FAIL ACLIDENT, REAR-END ELACK RCCK, NY 15 JUN 75 WAIL ALCIOEMT, KEAR-ENC ME YORK LITY, AY ZY JUL 75

FAIL DEKAILAENI, FIKE, EXPLUSIOA FEKTILE, MN 22 OCT 75

KAIL DEKAILNEN, FIKE, EXPLUSIOA FEKTILE, MN 32 OCT 75

FAIL ACLIUEAT, TEAD-UN COAHOMA, MS W TAVEN IN OCT 76

HAIL ACCICENT, HEAD-ON, C THAINS, NEW HAVEN IN OCT 76

KAIL ACCIDEAT, KEAR-END ENOOKLYA JUACTIUA WV 27 JAN 70

KAIL ACCILENT, KEAR-END SANEORN VY C 7 JAN 70

RAIL ACCIDENT, UEKAILREAT WARD AR 3 FEB 70

KAIL ACCIUEAT, KEAR-END TKACK CAK DINGLE ID 1 MAR 70

RAIL ACCIJENT, REAR-END ELAIRSVILLEPA 70 TAR 70

RAIL ACCIJENT, REAR-END ELAIRSVILLE PA $\quad 7$ APR 70

RAIL ACCIUEMT, ARTFAK DERAILMENT CMICAGU IL 15 UCT 70
RAIL ACLIDENT, GFADE CKUSDIIG GCLLEN MS

\begin{abstract}
RAIL ACLIDENT, GKADE CKUSDIVG GLLUEN, MS
\end{abstract}
(INPACT)

IMPACT, FENETRATIGN, SHIP CULLISIUN ANC GROUNDING

CMPACT, FENETRATICN, SHIP CULLISI

SS EAUNE COLLIDED WTAAKSMIP SS MEYTRADER IS JAN 74

$020<3-0 \Sigma \angle$ U U $29-L U 1$ CZCSZZ-JUj $0<4>4-3 U t$ $C 2 C=5-C 11$ $02625-611$

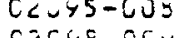
C<159-01c C2110-01C $0<118-004$ $021<0-005$ $021<1-\tilde{L} 4$ $021<2-304$ C21<3-005 C $<1<4-0 C s$ Cदि142-010 $02143-010$ $0 \overline{1} 1 \times 4-0 C_{4}$ C2155-0C5 $0,146-345$ $0<146-305$ C2149-0L4 C<2 $242-004$ $02203-000$

(IMFACI) $\cdots$

DEVERITIES UF TEANSFORTATIOP. ACCIDENTS VCL 1 SUMMARY

$015>0-001$ $015>0-001$
$017<5-001$ $021<6-001$
A1030-006 
CAFUC RESPOTSE TKUCK ANO RAIL ALCIDENTS

THAFSPOET ACCIUENTS OF KAR: $194 \bar{E}$ TU T9ES-UNITED KIVGLOM TKLCK ACCIDENTTELURIDA-C/C/71

TRULA ACLIDENTS, AHTSA, FIAAL REPUKT

TRUCK ACCIDENTI, BUTSA, VULC, NO. W

TKLCK ACCIUENTS, AHTSA, VULC,NO.5

TKUCK ACCIDLNTS, AHTSA, VCLC,NO.O

TRUCK ACCIUENTS, NHTSA, VULC, NO.7

TRUCK ACCIDENTS, NHTSA, VCLC, NO.8

TRUCK ACCIDEATS, NHTSA, VOLO, NO.Q

ACCIDENTS, CAMALA, KAM CCATAIMEKS

AUTO COLLISION WIANU CGLLAPSE UF DRIDGE, SILOAM, NC

TRUCK ACCIDENT, EXPLOSICN/FIRE, EAGLE PASS, TEXAS

SCHUULUUS/ALTO COLLISION/KOLLOVER, ASHLAND, OREGON

COLLISION UF TKUCK AND BUS, NEAK HAMILTUN, GA JUN 1975

COLLISIUR OF INAEBAGO MOTOK HOME WIERIOSE 1 JUL 1975

CCLLISIUN OF TKUCK AND CAR, LOVINGTON, NM 3 JAN 1976

LAR COLLISION, FEONT-TO-FRONT IPPACT TEST AT OC MPH

GASOLINE TRUCK ACCIUENT, SEATTLE, WA 4 DEC 1975

TRACTOF TKAILEN/CAR/SCHOOL OUS, REAR ENO, RULLOVEK

CAR EUS ACCIDENT, OVERLANL PAKK, KANSAS 7 OCT 1475

STATIUN WAGONITRAIN ACCIDENT, BALTIMORE, MD DEC 1975

IRACTOF TRAILEK, REAR EAD AND RCLLOVER NY 1 JUL 1975

TRUCK ALCIDENT, TIRE BLEW OUT, IMPACT, NY 21 JUN 1975

STATION WAGON/SCHOOL ELS, HEAL ON, HAMEURE, NY SEP 1975

TRUCK/SCHOOL BUSITRAIN ACLIDENT, AKKANSAS 11 SEP 1975

TRACTOR TRAILER, FRONT TO SIDE IMPACT TEO 1975

TRUCK CAR ACCIDENT INIERSECICN, SALTIMOKE,MD FEE 1975

CAR/POLE/TRAIA SIDESIIFE IMPACT,LUS ANGLES $\angle 5$ SEP 1975

TRUCK CAF ACCIUENT,KEAR END, SAN DIEOO FREEWAY JAN 1976

THUCK ACCIDENT, COLLISION LEFT KUADWAY ASHINGTON OC

TKUCK/VAA/AUTO ACCIDENT LEXINGTON VA O 20 AUG 1970

TFUCK, FECNT CORNEK IMFACT, CONCOKO, NY 27 NOV 1973

CAR/TRACTOR-TRAILER UNDERKIDE COLLISION 13 DEC 1974

TRUCK CAK ACCIDENT PACIFIC COAST HIGHWAY 17 JUA 1475

WHITE FREIGHTLINEK ACCIDENT VENTURA FREEWAY JUN 1975

TRUCK TKAILER ROLLOVEK, PUOE GRAKES, VERN .,CA JUN 1975

TANKTRUCK ACCIDENT, HELIUM FUEL LEAKAGE JUL 1975

TANKTRUCK ACCIDENT, HELIUM FUEL LEAKAGE

JACKNIFING IRAILER FORCED TKACTOR INTO BRIDGE JUL 1975
TRUCK TKAILER ACC IDENT, CARGO SHIFT, ROLLOVER AUG 1975

CAFITRAIN, GRADE CRLSSING, OES MOINES, IOWA SEE DE ZCOS

TRACTOR TRAILER COLLIDED WITH CAK DAXTER,CA 11 MAR 77

TRACTOR/CARLO TANK TRAILEK ACCIUENT, CHERRY HILL, NJ

TANKTKUCK/KAIL GRADE CRUSSINO ALCIDENT SEE DO $20 S 5$

SCHCOL GUS TRAIN GRADE CHUSSIAG ACCIDENT SEE DO COS8

TKACTOR-SEMITRAILER COLLISION MULTIPLE VEHICLES AUG 76

MIAI MOTCh HCME/CAR ACCILENT MCALESTER, OK 14 JUL 77

TKUCK ACCIDENT, FENETRATEC EKIDUE FAIL KELEASED HM CG

TAAKTKUCK RAIL CFUSDINE ACCIOENT SEL DBL11S 10 MAR 75
$01008-002$

Citog-C 11
0ite

(1) $275-014$

$01876-003$

Cic $77-2 \mathrm{C} 4$

$01878-508$

$41=79-c 06$

$01000-011$
$01801-014$

$01801-014$
$01008-013$

$01952-014$

$01953-011$

C1954-017

01955-009

C1456-015

C1457-000

$01958-002$

$01406-001$

$01967-C 05$

01908-005

01909-006

$01870-005$

$01 \times 70-005$

$01971-005$
$01972-005$

$01972-005$
$01973-005$

01974-006

01975-006

01976-004

C1977-005

C1977-C05

$02007-007$
$02013-007$

$02030-006$

$02033-004$

$02039-004$

02040-004

$02041-004$

$02043-004$

$02044-004$

$02045-004$

62007-001

02092-007

$020 \times 4-005$

02046-0C1

02047-001

$02112-C 13$

02115-014

02116-010

$02119-001$ 


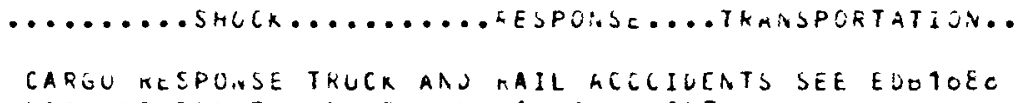

HAIL ACCIUENT, UNEUNTA, NY $1 \angle$ FLCE, 1974

KAIL ACCILENT, MUSTANG,OK, TSEMT, 1 \% 74

RAIL ACCIUENT, MEAD-IN, EL POOTE, CA 2 APE 1473

KAIL ACCIOENT, MEAD-ON, LUFKIN, TKXAS I8 NOV 1973

KAIL ACCIUENT, HEAD-ON, COEDEN, ILLINCIS 2 OEC 1973

KAIL ACCILENT, MEAD-ON, PLAIAVIE, TEXAS 15 DEC 1973

FAIL ACCILEAT, HEAD-ON, ST LUUIS,MISSOURI L2 JUN 1974

KAIL ACCICENT, HEAD-ON, MEMPHIS, TEN 24 JA. 1575

RAIL ACCIDENT, SIDE-ON, EARLS, TEAAS $\angle 9$ NON 1974

RAIL ACCIDENT, OPEN SWITCH, WINCHAM, GHIJ 22 NUV 1473

FAIL ACCIDENT, FAKING, LAKE JUNCTION, NJ 17 JUN 1474

FAIL ACCIOENT, FEAR END, HUKFICAIE, ALASKA 5 JUL 1975

RAIL ACCIDENT, REAR END, LEETONIA, OHIO 6 JUA 1975

KAIL ACCIDENT, REAR END, LUFG, ILLINOIS 3 JAV 1975

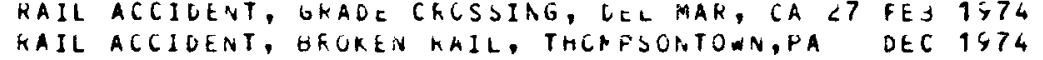

KAIL ACCIDENT, GKADE CKCSSINU, TFACY,CA 9 MAR 1575

KAIL ACCIOEITT, GKADL CRGSOIING, ELWOUD, IL IS NOV IY75

RAIL ACCIDENT, UAPRUTECTED GKADE CROSSING 7 FEO 1776

KAJL ACCIDENT, DEKAILMENT JAREATT VA

5 MAY 1970

KAIL ACCIDENT, MEAD-UN \& TRAIUS PETTISVILLE OH

ALTCHATED WHELL-OA-THE-GRCUND DEKAILMENT DETECTION SYS IIRMINGHAM OERAILEMENT, TKACK UROE IDENS 16 JAN 1977 KAIL CFOSSING ACCIDENT WITH TAAK TRUCK SEE EDOZCZO CAR/TRAIA-FRONT TO SIUE IRPACT UNPRUTECTEO CROSSING SCHOUL BUS-TRAIN COLLISION STRATTON, NE C AUG 1970 RAIL ACCIDENT, GFADE CRUSSING, DES MOINES, IOWA

RAIL ACIIUENT, GFADE CKOSSING, MAKLLAND, OK 15 DEC 76 KAIL CMOSSIAG ACCIDEAT. ITH SCHUUL GUS LAFAYETTE, OR TAILIN ACCIDENT, KAIL FAILURE, RELEASED ANHYDROUS AMMON TRAIN DERAILMEAT, FIKES AND EXPLUSIUN, OLLT, MONTANA KAIL CEUSSING ACCIDENT MANKTRUCK TRAYER, OS 1U MAK 7 TRAIN ACCILENT, CULLISIGR WIMAIL MAIN, AL 10 MIAR 75 RAIL ACCIUENT, REAR-ENC GLACK ROCK, AY 15 JUN 75 KAIL ACCIUENT, REAR-END NEW YORK CITY, NY i JUL 75 FAIL DEKAILMENT, FIRE, EXPLOSIUN HEKTILE, MN 22 UCT 75 RAIL ACCIDENT, HEADOON CUAROMA, MS 23 VOV 75 KAIL ACCIDENT, HEAO-ON, 2 TRAINS, NEN HAVEY IN UCT 70 RAIL ACCIOENT, DEKAILMENT COUGAAV YS AMTRAK SU JUN 76 GAIL ACCIDENT, REAR-ENC ZNCUKLYN JUNCTIUN WV 27 JAN 70 RAIL ACGIUENT, KEAR-ENO SANEOKN NY 27 JAN 70 RAIL ACCIDENT, DERAILNENT WARD AR 3 FEB 70 KAIL ACCIDENT, REAR-END TKACK CAK DINGLE IU 1 MAR 70 RAIL ACCILENT, REAR-END YLAIRJVILLE PA 7 APR 70
ธ1049-Cしட $013>\mathrm{C}-\mathrm{i} 1 \mathrm{C}$ $01592-015$
$01465-004$
$01426-003$ $014<6-003$ $01 \times<7-3<3$ $014<8-003$ $01768-304$ $01930-003$ $01951-002$ $01+32-0 C \bar{C}$ $01 \times 33-0 C 4$ $014.0-014$ $1 \times 47-0=4$ $01 \div 48-003$ $01 \times 48-003$
C1948-0U C1950-015 $01551-612$ CZC10-506 $42011-013$ OCO17-0C6 $0<0 \div 3-002$ $02029-001$ CCOC2-CŨS C2034-006 C2C55-000 CŹC8-009 $0<1<9-012$
$0<110-010$ C<118-CE4 $021<0-005$ $021<1-0<4$ $0<1<2-3<4$ C2124-J03 जC 142-01C $0<143-010$ $C 21 \times 4-C u 4$
$0<1>5-005$ $52150-200$ iट $1>7-0<5$ $C<1 \times 9-6) 4$ 


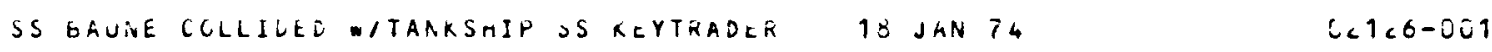

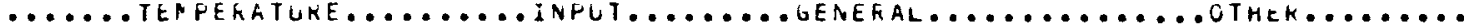

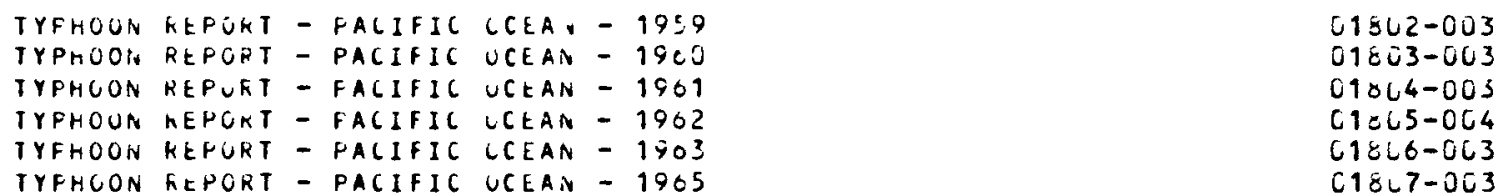

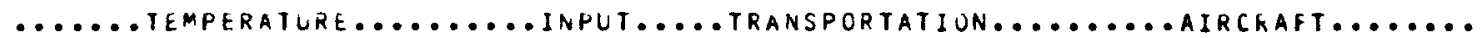

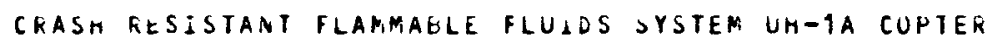

$02001-303$

(FIRE)

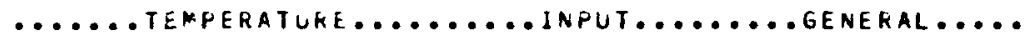

AIKCKAFT ACCIDENTS INVOLVING FIKES 1903-1974 ANALYSIS

$01940-002$ AIFCRAFT ACCIDENTS INVOLVING FIRES 1965-1974 SPECIAL

SEVEFITIES TRAN ACCIDENT VOL 2 CAKGO AIRCRAFT SEE 1030 CIVIL AVIATION ACCIOENT DATA - 1402 TO 1972

FIRE INFORMATICA AIRCRAFT ACCIDENTS

FIRE INFORMATICA AIRCRAFT ACCIDEN
AIC ACCIDENI MARTIN 4 O OCT 1970

A/C ACCIDENI MARTIN 4 C 4 OCT 1970
A/C ACCIDENT CUNLAIK SEC DEC 1905

A/C ACCIDENT CUNLAIK SEC DEC 1905
$A / C$ ACCIDENT DOUGLAS CS4D AFRIL 147U

AIC ACCIDENT 74712 SEPT 1970

A/C ACCIUENT DCO G3F SEFT 1470

AIPCKAFT ACCIDENT - BUEING $707-1110174$

AIRCRAFT ACCIDENT - DC C - O/20175

A/C CRASH, O7O7, BOSTUN, 3 IOUV 1473

A/C CRASH, O7C7, BOSTUN, 3 INUV 1473
AIRCRAFT ACCIDENT, DOEING $727-225$

AIRCKAFT ACCIDEAT, CONVAIN VT-Z9D $(C V-34 U)$ OS JAN 75

$19002-001$

$01004-009$

$01674-002$

$01674-002$
$01677-005$

$01677-005$
$01678-003$

$01600-063$

$01605-003$

$01006-003$

c1000-001

C1006-001

$018<1-001$
$01843-023$

$01843-023$
$0<152-059$

C.1>3-031 
MIKGMAFT ACCIUEST, LC-E CZ AIFCAAFT ACCIDENT, $C-C$ CZB AIKCKAFT ACLILENT, $\triangle C-4 \geq 1$

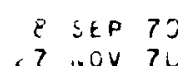

11 SEP 74

FIFE SAFETY EVALUATIOIV LF AIECRART LAFGO COMFARTIENT $\therefore<1,9-6 \leq 0$ $C<1<0-L+C$ L $2101-34$
(FIRE)

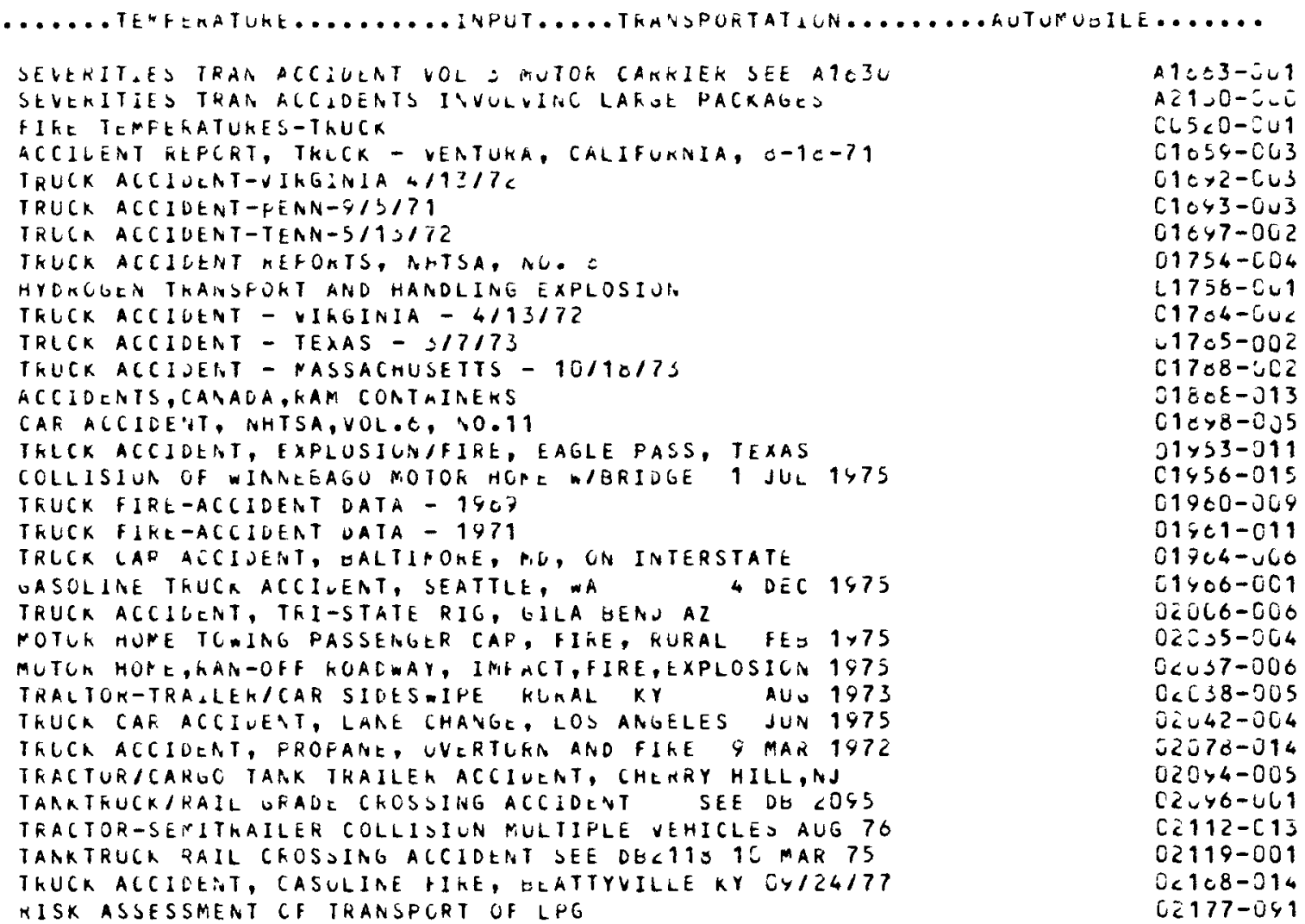

SEVERITAES TRAN ACCIUENT VOL Z MUTOK CAKKIEK SEE ATEZU SEVEKITIES TRAN ACCIDENTS ISVULVIRC LAKOE PACKAGES

FIKE TEMFERATUKES-THUCK

ACCILENT KEFCRT, TKLCK - VEATUKA. CALIFUKNIA, O-1C-71

TRUCK ACCIUCAT-VIKGINIA W/1?/7C

IRUCK ACCIDENT-FENN-G/3/71

IRLCA ACCIUENT-TEAN-5/13/72

TKUCK ACCILENT REFONIS, AHTSA, NG.

HYOKCOEN TKANSFOKT AND HANDLINE EXPLOSIUA.

TKLCK ACCIUENT - VIKGINIA - 4/13/72

TRLCK ACCIDENT - TEXAS - $\$ 17 / 73$

TKUCK ACCIDERT - NASSACHUSETTS - 10/10/7S

ACCIDENTS, CANAOA, KAM CONTAINEKS

CAF ACCIDE.TT NHTSA, YOLOO, TOO 11

IKLCK ACCJDEAT, EXPLUSIUNGFIRE, EAGLE PASS, TEXAS

COLLISIUA OF WIANEEAGU MOTOK HCPE IBRIDGE 1 JUL 1475

COLLISIUA OF WIAAEEAGU NOTOK HCRE

TKUCK FIRE-ACCIDENT DATA - 1907
TKUCK FIKE-ACCIOEAT OATA - 1971

TRLCK LAP ACCIDENT, OALTIPORE, PID, ON INTERSTATE

OASOLIAE TKUCK ACCIUEAT, SEATTLE, WA

TRUCK ACCILENT, TRI-STATE RIG, GILA BEAJ AZ

MOTUK MUVE TC.ING PASSEIVGER CAP, FIRE, RURAL FE 1975

MUTCN HORE, KAN-OFF KOACWAY, IMIACT, FIRE, EXPLOSICA 1975

TRALTOK-TRADLEKICAR SIOESIPE KLAAL KY AUD 1973

TKUCK CAF ACCIUEMT, LANE CHAVGE, LOS ANGELES JUN 1975

TKLCR ACCIDENT, FROFANE, UVERTLKA AND FIKE \& MAR 1972

TRACTUR/CAROC TAAK TRAILEK ACCIUENT, CHERRY HILL, NJ

TAAKTEUCK/RAIL URADE CKOSDING ACCIDEVT SEE DE COSS

TKAGTOR-SEITTKAILER COLLIDIUN MULTIPLE VEHICLED AUG 76

TAAKTRUCK RAIL CFOSDING ACCIDERT SEE DECIIS IC MAR 75

TKUCK ACCIOENT, CASULIAE HIKE, ELATTYVILLE KY GY/24/77

KISK ASSESSMENT CF TRANSPCRT OF LPG

A $1: \div 3-\Sigma \cup 1$

A $21,0-2-\mathrm{C}$

CL5 $\angle 0-2 \cup 1$

$01054-003$

$010 \times 2-\operatorname{Cus}$

$01043-003$

$01647-002$

$01647-002$

$01754-C 04$

$11758-C+1$

C1704-6ú
$1705-002$

C17 $178-i 02$

$0180 \varepsilon-313$

$01 \mathrm{c} \times 8-0 \mathrm{~J} 5$

$31 \times 53-011$

C1456-015

C1456-015

$01900-009$

$01>01-011$

$017 e 4-v 00$

¿1406-001

$0<006-000$

$02025-004$

Ccus $5-000$

$0<C \leqslant 8-905$

$02042-004$

52078-014

$020 \times 4-005$

$02+40-461$

Cद $112-C_{13}$

$02119-001$

$0<108-314$

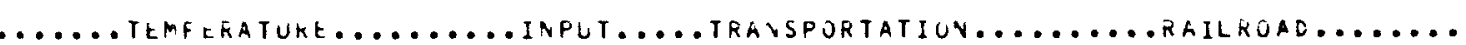

DEVERITIES TRAN ACCDUENT VOL \& TKAIN SEE A1CZO

SEVEKITIES NAIL ACCIUEATS LAKUE FACKAGLS SEE LO A213L

A $1900-\cup U 1$ FIKE TEMFEFATUKES-FAIL

KAIL ACCIDERT IANK CAR FUPTLRE, TIRE

IRANSPORT ALCDUEATS OF KAM 194:-1Y6S SEE EDS 1604

KAIL ACCIUEAT, CUTULLA, TEXAS - 1211/73

NAIL ACCIDENT, INOIU, CALIFURNIA - C/LS/73 
KAIL ACCIDENT, SALEM, ILLINCIS - C/10/71

KAIL ACCIDEINT, MAGUIN, ILLINOAS - $5 / 24 / 72$

KAIL ACLIDEAI IAFT LOUIOIANA - - IIC1/7

KAIL ACCIUEIVT, EAST ST LOUIS, ILLINCIS - 112217

KAIL ACCIOEWT, EAST ST LOUIS. ILLINCIS

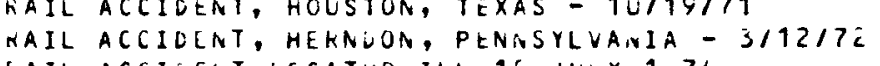

KAIL ACCIUEIVT, UECATUR, ILL IS JULY, $1 \times 74$

RAIL ACCIDENT, LNEUNTA,NY TE FEE, 1974

NAIL ACCIDENT, EENSON,AF 24 MAY, 1573

RAIL ACCIDENT, MILSTAIVG, ON, 1SEPT, 1Y74

TRAIA DEKAILMEIVT CAKRYING \& CUNTAINERS OF RAM

IAIL ACCIDENT, GRADE CKOSDING, MAKLAND, OK 15 UEC TE

TKAIN OERAILMEIOT, FIRES AND EXPLOSION, OELT, MONTANA

RAIL CROSSING ACCIDENT ITANKTRUCK THAYER, MS 10 MAR 7

RAIL CROSSING ACCIDENT ITANKTRUCK THAYER, MS 10 MAR 7
RAIL DERAILMENT, FIKE, EXPLOSION FERTILE, MN 22 OCT 75

RAIL DERAILMEAT, FIRE, EXPLOSION FERTILE, MN 220

RAIL ACCIDENT, DERAILMENTIFIRE CALVERT TX 30 MAR 76

$01763-062$
$01764-609$

$01764-601$

$01700-003$
$01700-010$

$01708-010$

RAIL ACCIDENT, DERAILMENT CLIFFURD MI 10 OCT $76 \quad 02201-003$

$017.9-003$

$01889-012$

$01890-012$

C1 $<41-010$

$02449-002$

$020 \times 5-008$

$02095-008$

0
$02118-004$

$02123-005$

$02147-014$

$021 \times 8-005$

FIRE TEMPERATUEES-SHIF

TANSHIP ACCIDENT, PENEYRATION/GAS FIRE IN CARGO TANKS

SS GAUNE COLLIDEL WITANKSHIF SS KEYTRADER 1E JAN 74

EXPLUSION FIFE ENGULFED THE MIT ELIAS, UNLOADING APR 74

RISK ASSESSMENT OF TRANSPURT OF LNG SEE EDE 2177

$00561-001$

$2048-001$

$021<6-001$

$02127-003$
$02178-001$

( $F I R E)$

..... temperature........ Input.... transport

$02178-001$

SEVEKITIES OF TKANSPORTATION ACCIDENTS VOL 1 SUMMARY

$11630-006$

( $I R E$ )

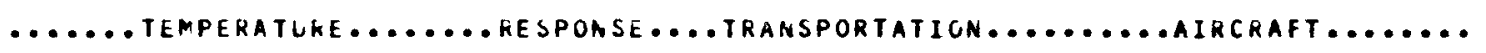

AIC CRASH, BTOT,EOSTUN, 3 NOV 1973

$01843-023$

( $F$ IRE)

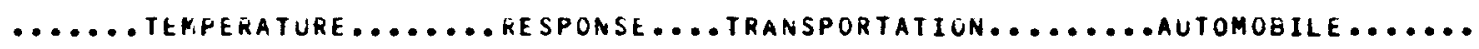

CARGO RESPONSE TRUCK AND KAIL ACCIDENTS

TRANSPORT ACCIDENTS OF RAM 1948 T0 1965-UNITED KINGDOM

ACCIDENTS, CANADA, RAM CONTAINERS

CAR ACCIDENT, NHTSA, VOL.O, NO.11

TRUCK ACCIDENT, EXPLOSION/FIRE, EAGLE PASS, TEXAS

COLLISION OF INAEEAGO MOTOR HUME W/BKIJGE, 1 JUL 1975

TRUCK CAR ACCIUENT, BALTIMOGE, MD, ON INTERSTATE

GASOLINE TRUCK ACCIUENT, SEATTLE, WA INTERSTATE
TRUCK ACCIDENT, TKI-STATE RIG, OILA BENDAZ

MOTCK HOME TCWINE PASSENGER CAF, FIRE, RURAL FEB 1975

MOTOR HURE, RAN-OFF KOADWAY, IMPACT,FIRE, EXPLOSION 1 T

TKACTOR-TRAILERICAR SIDESWIPE KUKAL KY AU 1973

01088-002

$01689-011$

$01888-013$

$01898-005$
$01953-011$

$01956-015$

$01404-306$

01906-001

$01906-001$
$02006-006$

$02006-006$
$12035-004$

C2037-000

C20 $28-005$ 
TKUCK CAR aCCIUED, LAPE CHARCE, LC: ARGELES JUV 1475 IKLCK ACCILLJT, PKOHANE, OVEKTUMI. ANO FIRE G MAK IYTC TFACTOK/CA2UO TARK TRAILEK ACCILETT, CHEKKY HILL, NJ TARKTKLCKIKAIL UKADE CRCSSIING ALCIDLIVT DEE DO CCYS IKACTOK-SEMITKAILER COLLIDICN MULTITLE VEHICLES AUG 70

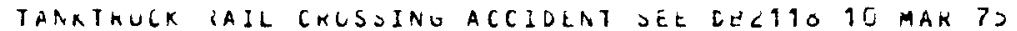

TKLCK ALCIUEAI, UASULITE FIRE, EEATTYVILLE KY UYIC4/77

$C \check{U}+2-C C$ $C<>>1 \varepsilon-01$ $C \leq ⿻>4-C C S$ JECYO- $=01$ ¿2112- 313 $\cup<119-001$ $c \bar{c} 10 \bar{c}-\tau 14$
( IIRE)

( $P \perp R E)$

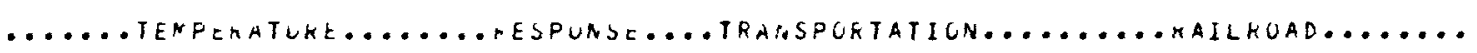

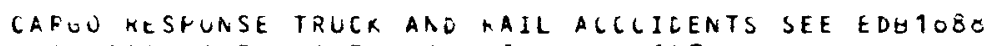

RAIL ACLIDEIVT, DECATUK, ILL 1Y JULY, 1S74

FAIL ACGIUENT, UNEOATA,WY 12 FEE, 1974

NAIL ACCIUENT, OEESON,AK 24 MAY, 1973

KAIL ACCIDETOT, MUSIATU, UK, ISEPT, TY74

TKAIN DEKAILFEAT CAKRYING Ć CUNTAINERS UF RAF

TKAIL ACCIDENT, GRADE CKOSSING, MAFLANO, OK IS DEC 70

TRAII OERAILMENT, FIKES ANO EXPLCSICN, ELLT, MONTANA

KAIL CKUSSIINC ACCIDENT WITANKTRUCK THATEK, MS IC MAR

RAIL UEKAILPENT, FIKE, EXFLUSICA FEKTILE, MA 22 ULT TS

RAIL ACCIDENT, DEKAILMENT GULOCNNA LA CO DEC 77

RAIL ACCIDENT, CLEAILMENTIFIRE CALVERT TX 30 MAR 76

し1०४จ-0U2

$01009-012$

$015 \times 0-51<$

$01841-010$

$018 \times 2-010$

$0<049-002$

MAIL ACCIDENT, DLAAILMENT CLIFFOKD F

18 CCT 70

$C<110-010$

Cटि118-Cu4

$0<1<3-605$

Q2 $147-314$

$C 2148-i C 5$

$0<\angle 01-003$

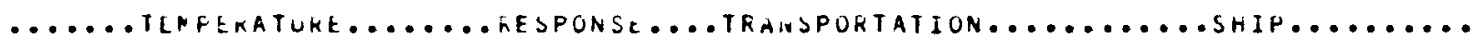

TANSHIP ACCIDENT, FENETRATION/GAS FIKE IN CARGO TANKS SS EAUNE COLLILEO ITANKSHIP SS KEYTKADER 1O JAN 74
EXPLCSION FIFE ENGLLFED THE MIT ELIAS, UIVLOADING APR 74

$C=04 \hat{\theta}-C 01$ $C 2126-001$

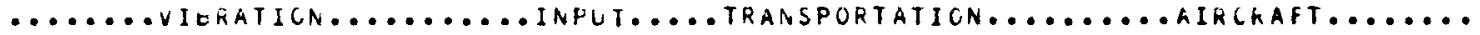

AIFCKAFT ACCIDENT, UH-114 11 JUL $75 \quad 021 C 3-036$ .......... Int............ INPLt....... General...........

TKOF ICAL GYLUNES

$01556-042$

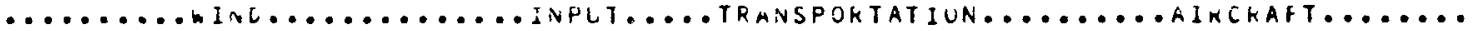

MELICUPTER DOWVASH DATA

C17ยC-iう1

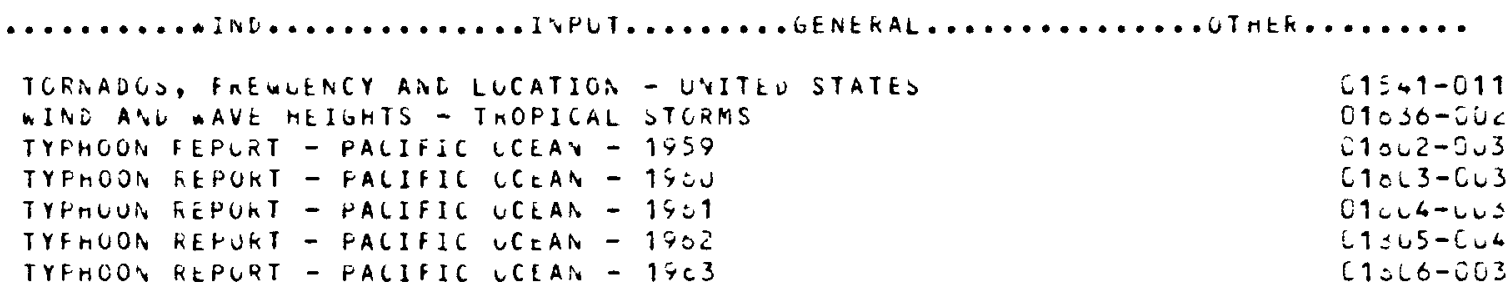


$\left(S I C R M_{1}\right)$

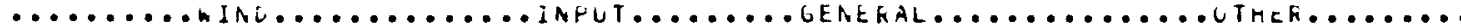

TYFHCON KEFUKT - HACIFIC UCEAIV - 19CS

TYPHUGQ FEHOHI - FACIFIC UCEAM - $19 C 0$

TYPHCON, KE

TYPHCON REPURT - PACIFIC UCCAN - 1908

TYFHOUN REFURT - FALIFIC UCEAN - 1909

IYPHOON KEPURT - PACIFIC CCEAN - 1970

TYPHGON REFURT - PACIFIC UCEAIV - 1974

C1: $17-003$

$01008-5 \cup 3$

01609-003

$1010-004$

$01611-00 \hat{<}$

$01 \varepsilon 12-003$

( $S$ TURM)

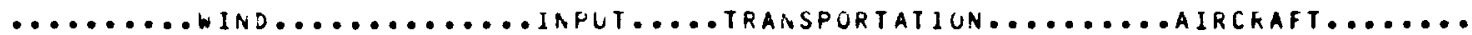

AIRGRAFT ACEIDENT, BOEING $727-2 \angle 4 \quad 7$ AUG 75

C $155-047$ 
DISTRIBUTION

TID-4500 R66, UC-71 (171)

Transportation Branch (5)

DOE

Division of Environmental Control Technology

Washington, DC 20545

National Science Foundation

2101 Constitution Avenue, NW

Washington, DC 20418

Attn: W. M. Carey

A. Grella (3)

DOT

Office of Hazardous Materials

Room 6218, Buzzard's Point Bullding

2100 2nd SW

Washıngton, DC 20590

L. Benner

Chief

Hazardous Materials Safety Division

NTSB

Washington, DC 20594

J. Russell

Environmental Protection Agency

Office of Radiation Programs

401 M Street, SW

Washington, DC 20460

W. F. Black

DOT

Federal Rail Administration

Trans Point Bullding

Washington, DC 20590

J. Buchanan

Nuclear Safety Information Center

P. O. Box $Y$

Oak Ridge, TN 37830

R. E. Kidwell

DOT

Federal Highway Administration

DOT Bullding, Room 3408

Washington, DC 20590

W. E. Scott

National Highway Traffic Safety Administration

Office of Accident Investigation

and Data Analysis

Nassif Bullding

4007 th Street, SW

Washington, DC 20590

Mr. S. G. Guins

4496 Doble Road

Okemos, MI 48864
Atso Savisaar
A RINC Research Corp.
2551 Riva Road
Annapolis, MD 21401

R. Hall

Battelle Pacific NW Laboratories

Nuclear Technology Dept.

Batelle Blvd

Richmond, WA 99352

George H. Kinchin, Director

Safety \& Reliability Directorate

United Kingdom Atomic Energy Authority

Wigshaw Lane

Culcheth Warrington, Cheshire, England

WA $34 \mathrm{NE}$

Bell Laboratories, Inc

Whippany Road

Whippany, NJ 07981

Attn: Marian Rello

RM 5 E-227

Dr. Hartwig

Battelle - Germany

Instutut $\mathrm{E} . \mathrm{V}$.

Postfach 900-160

D-6000 Frankfurt 90

Deutchland (FRG)

Henry Pusey, Director

Shock and Vibration Information Center

Naval Research Lab

Code 8404

Washington, DC 20375

S. R. Flelds

Hanford Engineering Development Laboratory

Westinghouse Hanford Company

Box 1970

Richland, WA 99352

Los Alamos Scientıfic Laboratory

P. O. Box 1663

Los Alamos, NM 87544

Attn* Report Librarian

S. F. Petry

E. I. du Pont de Nemours \& Company

Savannah River Laboratory

Alken, SC 29801

U. S. Nuclear Regulatory Commissıon

Division of Safeguards

Washington, DC 20555

Attn W. Lahs

U. S. Nuclear Regulatory Commission

Office of Standards Development

Washington, DC 20555

Attn D. J. Guzy 
DISTRIBUTION: (Continued)

R. J. Phelan

Director of Technical Committees

Association of American Railroads

1920 L Street, NW

Washington, DC 20036

D. R. Ahlbeck

Battelle Columbus Laboratories

505 King Avenue

Columbus, $\mathrm{OH} 43201$

G. D. Bell

United Kingdom Atomic Energy Authority

Wigshaw Lane

Culcheth Warrington, Cheshire, England

WA $3 \quad 4$ NE

L. T. Wilson

506 Dartmouth SE

Albuquerque, NM 87106

$\begin{array}{ll}1000 & \text { G. A. Fowler } \\ 1500 & \text { W. A. Gardner } \\ 1700 & \text { W. C. Myre } \\ 1710 & \text { V. E. Blake, Jr. } \\ 4300 & \text { R. L. Peurifoy, Jr. } \\ 4400 & \text { A. W. Snyder } \\ 4442 & \text { W. A. Von Riesmann } \\ 4500 & \text { E. H. Beckner } \\ 4530 & \text { R. W. Lynch } \\ 4541 & \text { L. W. Scully } \\ 4550 & \text { R. M. Jefferson } \\ 4551 & \text { R. E. Luna } \\ 4552 & \text { R. B. Pope } \\ 5500 & \text { O. E. Jones } \\ 5520 & \text { T. B. Lane } \\ 5522 & \text { J. T. Foley (15) } \\ 5522 & \text { T. G. Priddy } \\ 5523 & \text { C. A. Davidson (15) } \\ 5523 & \text { R. C. Reuter, Jr. } \\ 8121 & \text { G. W. Benedetti } \\ 8122 & \text { C. A. Scott (2) } \\ 8266 & \text { E. A. Aas } \\ 3141 & \text { T. L. Werner (5) } \\ 4151 & \text { W. L. Garner (3) } \\ & \text { For DOE/TIC (Unlimited Release) }\end{array}$

